This is a self-archived version of an original article. This version may differ from the original in pagination and typographic details.

Author(s): Kankainen, Anu; Novikov, Yuri; Schatz, H.; Weber, Christine

Title: Mass measurements of neutron-deficient nuclei and their implications for astrophysics

Year: 2012

Version: Accepted version (Final draft)

Copyright: @ S SIF, Springer-Verlag Berlin Heidelberg 2012

Rights: In Copyright

Rights url: http://rightsstatements.org/page/InC/1.0/?language=en

Please cite the original version:

Kankainen, A., Novikov, Y., Schatz, H., \& Weber, C. (2012). Mass measurements of neutrondeficient nuclei and their implications for astrophysics. European Physical Journal A, 48(4), 50. https://doi.org/10.1140/epja/i2012-12050-5 


\title{
Mass measurements of neutron-deficient nuclei and their implications for astrophysics
}

\author{
A. Kankainen ${ }^{1 \mathrm{a}}$, Yu. N. Novikov ${ }^{2,3}$, H. Schatz ${ }^{4}$, and C. Weber ${ }^{5}$ \\ 1 Department of Physics, University of Jyväskylä, P.O. Box 35, FI-40014 University of Jyväskylä, Jyväskylä, Finland \\ 2 Petersburg Nuclear Physics Institute, 188300 Gatchina, Russia \\ 3 GSI Helmholtzzentrum für Schwerionenforschung GmbH, Planckstrasse 1, D-64291 Darmstadt, Germany \\ 4 Department of Physics and Astronomy, National Superconducting Cyclotron Laboratory, and Joint Institute for Nuclear \\ Astrophysics, Michigan State University, East Lansing, Michigan 48824, USA \\ 5 Faculty of Physics, Ludwig-Maximilians-Universität München, Am Coulombwall 1, D-85748 Garching, Germany
}

Received: date / Accepted: date

\begin{abstract}
During the years 2005-2010 the double Penning trap mass spectrometer JYFLTRAP has been used to measure the masses of 90 ground and 8 isomeric states of neutron-deficient nuclides with a typical precision of better than $10 \mathrm{keV}$. The masses of 14 nuclides $-{ }^{84} \mathrm{Zr},{ }^{88,89} \mathrm{Tc},{ }^{90-92} \mathrm{Ru},{ }^{92-94} \mathrm{Rh},{ }^{94,95} \mathrm{Pd}$, ${ }_{106,108,110} \mathrm{Sb}$ - have been experimentally determined for the first time. This article gives an overview on these measurements and their impact on the modeling of the astrophysical $r p$-process.
\end{abstract}

PACS. 21.10.Dr Binding energies and masses - 26.30.Ca Explosive burning in accreting binary systems (novae, x-ray bursts)

\section{Introduction}

Very neutron-deficient nuclei are synthesized in astrophysical environments, where extreme temperatures and densities as well as an abundance of hydrogen can lead to a rapid proton capture process ( $r p$-process) $[1,2]$. As proton capture rates become faster than $\beta^{+}$decays, unstable neutron deficient nuclei are built up. The most common occurrence of such a scenario are type I x-ray bursts, which occur on the surface of neutron stars that accrete hydrogen-rich matter from a companion star in a stellar binary system. The hydrogen-rich fuel layer on the neutron star surface builds up for hours to days before it explodes giving rise to a bright x-ray burst typically lasting for $10-100$ seconds. Such bursts are frequently observed with modern x-ray observatories and are directly powered by the nuclear energy generated in the $r p$-process reaching nuclei up to tellurium [3-5].

Recently, a new astrophysical process with rapid proton captures on neutron deficient nuclei has been proposed, the $\nu p$-process [6-8]. It takes place in supernovae and possibly in gamma-ray bursts where proton-rich outflows are created by strong neutrino fluxes [6]. In the $\nu p$ process, the flow towards heavier elements is accelerated via fast $(n, p)$ reactions on long-lived nuclides along the path of the $r p$-process. The neutrons that induce these reactions are created via antineutrino absorptions on protons. It has been shown that the reaction sequence can

\footnotetext{
a Email:anu.k.kankainen@jyu.fi
}

reach nuclei up to $A=108$ or $A=152$ depending on the electron fraction [9].

Nova explosions are a third astrophysical environment where a mild form of the $r p$-process takes place. They occur when an accreted fuel layer on the surface of a white dwarf star explodes. The $r p$-process in novae typically proceeds near stability and is predicted to end in the $A=40$ region [10-13], though recent model calculations have identified parameters that can lead to much more violent explosions with a stronger $r p$-process reaching the iron region [14]. Accurate nuclear physics is needed to predict the contributions of novae and the $\nu p$-process to Galactic nucleosynthesis, to interpret x-ray burst light curves in terms of neutron star properties and to predict the composition of the ashes of x-ray bursts needed to model neutron star crusts. With accurate nuclear physics one can also predict new observables that observational programs can search for.

Nuclear masses play a central role in rapid proton capture processes $[3,15-21]$. When proton capture rates are high, it is photodisintegration that limits further proton captures and forces the reaction sequence to proceed via a $\beta^{+}$decay or a $(n, p)$ reaction. Such photodisintegration rates depend exponentially on the energy required to remove a proton from the nucleus, and therefore on the nuclear masses. In addition, many of the important proton capture rates in the $r p$-process are governed by resonances. Rates depend exponentially on resonance energies, which in most cases are determined by measuring excitation en- 
ergies and nuclear masses. Typically, a precision of around $10 \mathrm{keV}$ is required, for some resonant reaction rates even much better than $10 \mathrm{keV}$ [16]. Penning trap mass measurements have had a major impact on nuclear astrophysics, as they can easily reach or surpass such precision even for the most unstable nuclei within reach. This is illustrated in Fig. 1, where Penning trap mass measurements since the Atomic Mass Evaluation 2003 (AME03) are highlighted. Clearly, the Penning trap approach has addressed the long standing problem of unreliable and uncertain experimental masses of unstable nuclei and has been applied to a large number of neutron-deficient nuclei close to or in the $r p$-process. In some cases, the effort to push to the most exotic nuclei has led to the paradox situation that now some of the masses of exotic isotopes are better known than their more stable counterparts. The program to measure masses of unstable nuclei with JYFLTRAP has been a major contributor to this success together with CPT [22] at ANL, ISOLTRAP [23] at CERN-ISOLDE, LEBIT [24] at NSCL, and SHIPTRAP [25] at GSI.

In addition to nuclear astrophysics, neutron-deficient nuclei offer a wealth of other interesting physics phenomena to be studied. For example, $Q_{\mathrm{EC}}$ value measurements at JYFLTRAP have been useful for testing the isobaric multiplet mass equation $[26,27]$ and the Conserved Vector Current (CVC) hypothesis [28]. Masses of neutrondeficient nuclei provide also important data for developing and testing different mass models. Energy systematics, such as two neutron or proton separation energies, yield information e.g. on the possible onset of deformation and the evolution of shell-gap energies as well as on neutronproton pairing and the Wigner energy [29].

\section{Experimental methods}

The ions of interest have been produced with beams from the K-130 cyclotron of the JYFL Accelerator Laboratory impinging on a thin (few $\mathrm{mg} / \mathrm{cm}^{2}$ ) target situated at the Ion-Guide Isotope Separator On-Line (IGISOL) facility [30]. Which ion guide is used depends on the applied beam: a heavy primary beam would cause plasma effects inside the gas cell and thus it is stopped before entering the gas cell whereas light beams, such as $p$ or ${ }^{3} \mathrm{He}$, can pass through the gas cell. In the light-ion ion guide, the target is inside the IGISOL gas cell, where the product recoils are stopped, and a good fraction of the ions end up with charge state $1^{+}$. In the heavy-ion ion guide, also known as HIGISOL [31,32], the target is in front of the gas cell and the products have to recoil at small angles in order to pass through a Havar window around the beam stopper before thermalizing in the gas cell. The employed production methods are summarized in Table 1.

After thermalization, the ions are extracted from the ion guide with the help of differential pumping and an electric field. The ions are accelerated to $30 \mathrm{keV}$ and massseparated by a dipole magnet before entering the radiofrequency quadrupole (RFQ) [42], which is used for cooling and bunching of the ions. After the RFQ, the ions are injected into the JYFLTRAP [43] cylindrical double Penning trap situated inside a $7 \mathrm{~T}$ superconducting solenoid. The first trap is called the purification trap since it is used for isobaric purification via mass-selective buffer gas cooling [44]. The precision mass measurements are carried out in the second trap, known as the precision trap, via the time-of-flight ion cyclotron resonance (TOF-ICR) method $[45,46]$.

In the TOF-ICR method, the cyclotron frequency of the ion of interest with mass $m$ and charge $q$ in a magnetic field $B, \nu_{c}=q B /(2 \pi m)$, is compared to the cyclotron frequency of a reference ion $\left(\nu_{\text {ref }}\right)$ with a well-known atomic mass $m_{\text {ref }}$. Since the mass-separated ions have typically the same charge state of $1^{+}$at IGISOL, the mass of the ion of interest is obtained as: $m=\left(\nu_{\text {ref }} / \nu_{c}\right)\left(m_{\text {ref }}-m_{\mathrm{e}}\right)+m_{\mathrm{e}}$. Each measurement of the ion of interest is sandwiched between two reference measurements in order to determine the value of the magnetic field $B$ at the time of the actual measurement. Since the IGISOL method provides a large variety of possible reference ions to be measured on-line, the ion with a superior precision closest to the ion of interest is typically chosen for reference. In some cases, the production rate of oxides can be higher or comparable to the rate of the ions. For example, the masses of neutrondeficient yttrium, niobium and zirconium isotopes have been measured as oxides.

The data have been fitted and analysed mainly with the programs LAKRITSI and COMA [28]. A count-rateclass analysis [47] has been performed whenever possible. The uncertainty contribution of possible magnetic field fluctuations has been added, and mass-dependent and residual uncertainties have been included in the final atomic mass values. A detailed description of the data analysis can be found in Ref. [19]. The latest results for the mass-dependent and residual uncertainties applicable to the measurements performed after the June 2007 magnet quench are reported in the JYFLTRAP paper on carboncluster cross-reference measurements [48].

\section{Mass measurements of neutron-deficient nuclides at JYFLTRAP}

Measurements performed at JYFLTRAP in 2005-2010 have yielded 90 published ground-state and 8 published isomericstate masses of neutron-deficient nuclides (see Fig. 1). These measurements have been motivated for example by the astrophysical $r p$ - and $\nu p$-processes $[3,19,38,40,41]$ or nucleosynthesis in novae $[26,27,33]$ or isospin symmetry studies. The results of mass measurements related to the superallowed $0^{+} \rightarrow 0^{+} \beta$ decays $[33-37,39]$ are not discussed in this review. In the following, the measured masses are discussed from lighter to heavier nuclides and related energy systematics $\left(S_{\mathrm{p}}, Q_{\alpha}\right.$, and $S_{2 \mathrm{p}}$ values) are reviewed. The astrophysical implications are discussed separately in Section 4. 
Table 1. The production methods of the neutron-deficient nuclides measured at JYFLTRAP. LI stands for the light-ion guide and HI for the heavy-ion ion guide technique.

\begin{tabular}{|c|c|c|c|c|c|}
\hline$E_{\text {beam }}$ & Beam & Target & Ion-guide & Measured nuclides & Ref. \\
\hline $40 \mathrm{MeV}$ & $\mathrm{p}$ & ${ }^{\text {nat }} \mathrm{Mg}$ & LI & ${ }^{23} \mathrm{Al},{ }^{23} \mathrm{Mg}$ & {$[26]$} \\
\hline $35 \mathrm{MeV}$ & $\mathrm{p}$ & ${ }^{27} \mathrm{Al}$ & $\mathrm{LI}$ & ${ }^{26} \mathrm{Si},{ }^{26} \mathrm{Al},{ }^{26} \mathrm{Al}^{\mathrm{m}}$ & {$[33]$} \\
\hline $40 \mathrm{MeV}$ & $\mathrm{p}$ & $\mathrm{ZnS}$ & LI & ${ }^{31} \mathrm{~S}$ & {$[27]$} \\
\hline $15 \mathrm{MeV}$ & $\mathrm{p}$ & $\mathrm{ZnS}$ & LI & ${ }^{34} \mathrm{Cl},{ }^{34} \mathrm{Cl}^{\mathrm{m}}$ & {$[34]$} \\
\hline $20 \mathrm{MeV}$ & $\mathrm{p}$ & $\mathrm{KCl}$ & LI & ${ }^{38} \mathrm{~K},{ }^{38} \mathrm{~K}^{\mathrm{m}}$ & {$[34]$} \\
\hline $20 \mathrm{MeV}$ & ${ }^{3} \mathrm{He}$ & ${ }^{\text {nat }} \mathrm{Ca}$ & LI & ${ }^{42} \mathrm{Sc},{ }^{42} \mathrm{Sc}^{\mathrm{m}}$ & {$[35]$} \\
\hline $17 \mathrm{MeV}$ & ${ }^{3} \mathrm{He}$ & ${ }^{\text {nat }} \mathrm{Ca}$ & LI & ${ }^{42} \mathrm{Ti}$ & {$[36]$} \\
\hline $20 \mathrm{MeV}$ & $\mathrm{p}$ & ${ }^{46} \mathrm{Ti}$ & LI & ${ }^{46} \mathrm{~V}$ & {$[35]$} \\
\hline $13-15 \mathrm{MeV}$ & $\mathrm{p}$ & ${ }^{50} \mathrm{Cr}$ & LI & ${ }^{50} \mathrm{Mn},{ }^{50} \mathrm{Mn}^{\mathrm{m}}$ & {$[37]$} \\
\hline $40 \mathrm{MeV}$ & $\mathrm{p}$ & ${ }^{54} \mathrm{Fe}$ & LI & ${ }^{53} \mathrm{Co},{ }^{53} \mathrm{Co}^{\mathrm{m}}$ & {$[38]$} \\
\hline $13-15 \mathrm{MeV}$ & $\mathrm{p}$ & ${ }^{54} \mathrm{Fe}$ & LI & ${ }^{54} \mathrm{Co},{ }^{54} \mathrm{Co}^{\mathrm{m}}$ & {$[37]$} \\
\hline $105 \mathrm{MeV}$ & ${ }^{20} \mathrm{Ne}$ & ${ }^{\text {nat }} \mathrm{Ca}$ & $\mathrm{HI}$ & ${ }^{56} \mathrm{Co}$ & {$[38]$} \\
\hline $25 \mathrm{MeV}$ & ${ }^{3} \mathrm{He}$ & ${ }^{54} \mathrm{Fe}$ & LI & $55,56 \mathrm{Ni}$ & {$[38]$} \\
\hline $75 \mathrm{MeV}$ & ${ }^{20} \mathrm{Ne}$ & ${ }^{n a t} \mathrm{Ca}$ & $\mathrm{HI}$ & ${ }^{57} \mathrm{Ni}$ & {$[38]$} \\
\hline $40 \mathrm{MeV}$ & $\mathrm{p}$ & ${ }^{58} \mathrm{Ni}$ & LI & ${ }^{56} \mathrm{Ni},{ }^{57,58} \mathrm{Cu}$ & {$[38]$} \\
\hline $25 \mathrm{MeV}$ & ${ }^{3} \mathrm{He}$ & ${ }^{58} \mathrm{Ni}$ & LI & $59,60 \mathrm{Zn}$ & {$[38]$} \\
\hline $48 \mathrm{MeV}$ & $\mathrm{p}$ & ${ }^{64} \mathrm{Zn}$ & LI & ${ }^{62} \mathrm{Cu},{ }^{62} \mathrm{Zn},{ }^{62} \mathrm{Ga}$ & {$[39]$} \\
\hline $150-170 \mathrm{MeV}$ & ${ }^{3} \mathrm{~S}$ & ${ }^{54} \mathrm{Fe}$ & $\mathrm{HI}$ & $80-82 \mathrm{Y}$ & {$[40]$} \\
\hline $150-170 \mathrm{MeV}$ & ${ }^{32} \mathrm{~S}$ & ${ }^{\text {nat }} \mathrm{Ni}$ & $\mathrm{HI}$ & ${ }^{83} \mathrm{Y},{ }^{83-86,88} \mathrm{Zr},{ }^{85-88} \mathrm{Nb}$ & {$[40]$} \\
\hline $222 \mathrm{MeV}$ & ${ }^{36} \mathrm{Ar}$ & ${ }^{\text {nat }} \mathrm{Ni}$ & $\mathrm{HI}$ & ${ }^{84} \mathrm{Y},{ }^{87} \mathrm{Zr},{ }^{89} \mathrm{Mo},{ }^{88} \mathrm{Tc}$ & {$[19]$} \\
\hline $189 \mathrm{MeV}$ & ${ }^{40} \mathrm{Ca}$ & ${ }^{\text {nat }} \mathrm{Ni}$ & $\mathrm{HI}$ & ${ }^{88} \mathrm{Mo},{ }^{92} \mathrm{Tc},{ }^{91,94} \mathrm{Ru},{ }^{94-95} \mathrm{Rh}$ & {$[19]$} \\
\hline $205 \mathrm{MeV}$ & ${ }^{40} \mathrm{Ca}$ & ${ }^{\text {nat }} \mathrm{Ni}$ & $\mathrm{HI}$ & ${ }^{91} \mathrm{Tc},{ }^{91} \mathrm{Ru}$ & {$[19]$} \\
\hline $220 \mathrm{MeV}$ & ${ }^{40} \mathrm{Ca}$ & ${ }^{\text {nat }} \mathrm{Ni}$ & $\mathrm{HI}$ & ${ }^{89-90} \mathrm{Tc},{ }^{90,92-93} \mathrm{Ru},{ }^{92-94} \mathrm{Rh}$ & {$[19]$} \\
\hline $170 \mathrm{MeV}$ & ${ }^{40} \mathrm{Ca}$ & ${ }^{\text {nat }} \mathrm{Ni}$ & $\mathrm{HI}$ & ${ }^{94-96} \mathrm{Pd},{ }^{95} \mathrm{Pd}^{\mathrm{m}}$ & {$[19]$} \\
\hline $70 \mathrm{MeV}$ & ${ }^{3} \mathrm{He}$ & ${ }^{\text {nat }} \mathrm{Ru}$ & LI & $97-98 \mathrm{Pd}$ & {$[41]$} \\
\hline $62 \mathrm{MeV}$ & $\mathrm{p}$ & ${ }^{106} \mathrm{Cd}$ & LI & $99,{ }^{101} \mathrm{Pd},{ }^{101-105} \mathrm{Cd},{ }^{102} \mathrm{In}$ & {$[41]$} \\
\hline $70 \mathrm{MeV}$ & ${ }^{3} \mathrm{He}$ & ${ }^{106} \mathrm{Cd}$ & LI & ${ }^{104} \mathrm{In}$ & {$[41]$} \\
\hline $100 \mathrm{MeV}$ & ${ }^{3} \mathrm{He}$ & ${ }^{106} \mathrm{Cd}$ & LI & ${ }^{100} \mathrm{Ag}$ & {$[41]$} \\
\hline $295-330 \mathrm{MeV}$ & ${ }^{58} \mathrm{Ni}$ & ${ }^{\text {nat }} \mathrm{Ni}$ & HI & $\begin{array}{l}{ }^{104-108} \mathrm{Sn},{ }^{106-110} \mathrm{Sb}, \\
{ }^{108-109} \mathrm{Te},{ }^{111} \mathrm{I}\end{array}$ & {$[3]$} \\
\hline
\end{tabular}

\subsection{Mass measurements at $A=23$ and $A=31$}

The mass measurements at $A=23$ and $A=31$ have been motivated by nova nucleosynthesis [12] and the Isobaric Multiplet Mass Equation (IMME) (see e.g. Ref. [49,50]). According to the IMME, the masses of members with an isospin projection $T_{Z}=(N-Z) / 2$ of an isobaric multiplet $T$ should lie along a parabola $M\left(T, T_{Z}\right)=a+b T_{Z}+c T_{Z}^{2}$. In general, the IMME has worked well and it has been used to predict masses of more exotic members of the multiplet. A breakdown of the IMME could be explained for example by higher-order perturbations or by the importance of including three-body terms. In addition, a significant component of isospin mixing could also result in deviations from the quadratic behaviour [51].

At JYFLTRAP, the masses of ${ }^{23} \mathrm{Al}$ and ${ }^{23} \mathrm{Mg}$ have been determined precisely against the reference nuclide ${ }^{23} \mathrm{Na}$. The new mass value for ${ }^{23} \mathrm{Al}$ is $22(19) \mathrm{keV}$ lower and 55 times more precise than the AME03 [52] value. The JYFLTRAP mass value for ${ }^{23} \mathrm{Mg}$ agrees with the AME03 value [52] and almost doubles the precision. The most recent ground-state masses for ${ }^{23} \mathrm{Al}[26],{ }^{23} \mathrm{Mg}[26$, 52 ], ${ }^{23} \mathrm{Na}$ [52] and ${ }^{23} \mathrm{Ne}$ [52], and excitation energies for the $T=3 / 2$ isobaric analog states $[53,54]$ were adopted and a quadratic IMME fit $\left(\chi^{2} / n=0.28\right)$ was performed for the $T=3 / 2$ quartet at $A=23$ in Ref. [26]. The cubic fit yielded a cubic term $d=0.22(42) \mathrm{keV}$ consistent with zero. Thus, the IMME was found to work well in the $A=23$ quartet.

Another interesting $T=3 / 2$ quartet lies at $A=31$ : ${ }^{31} \mathrm{Si},{ }^{31} \mathrm{P},{ }^{31} \mathrm{~S}$, and ${ }^{31} \mathrm{Cl}$. For this quartet, the mass of ${ }^{31} \mathrm{~S}$ has been precisely measured at JYLFTRAP and was found to deviate from the AME03 value [52]. Unfortunately, an attempt to measure the mass of the much more uncertain ${ }^{31} \mathrm{Cl}$ nucleus failed due to overwhelming nitrogen oxide background at $A=31$ caused by poor vacuum conditions in the IGISOL facility during that run. In the future, a mass measurement of ${ }^{31} \mathrm{Cl}$ would be very interesting at the new IGISOL-4 facility.

Currently, the only isobaric quintet that has been measured precisely enough to test the IMME lies at $A=32$. Although there have been no mass measurements at $A=$ 32 at JYFLTRAP, the IMME at $A=32$ has been studied in one of our publications [27]. A recent ${ }^{32} \mathrm{~S}\left({ }^{3} \mathrm{He}, \mathrm{t}\right){ }^{32} \mathrm{Cl}$ measurement [55] showed a discrepancy to the ${ }^{32} \mathrm{Cl}$ mass obtained from the proton separation energy [56] and the ${ }^{31} \mathrm{~S}$ mass from AME03. The mass of ${ }^{31} \mathrm{~S}$ measured at JYFLTRAP was found to deviate from AME03 [52] by 


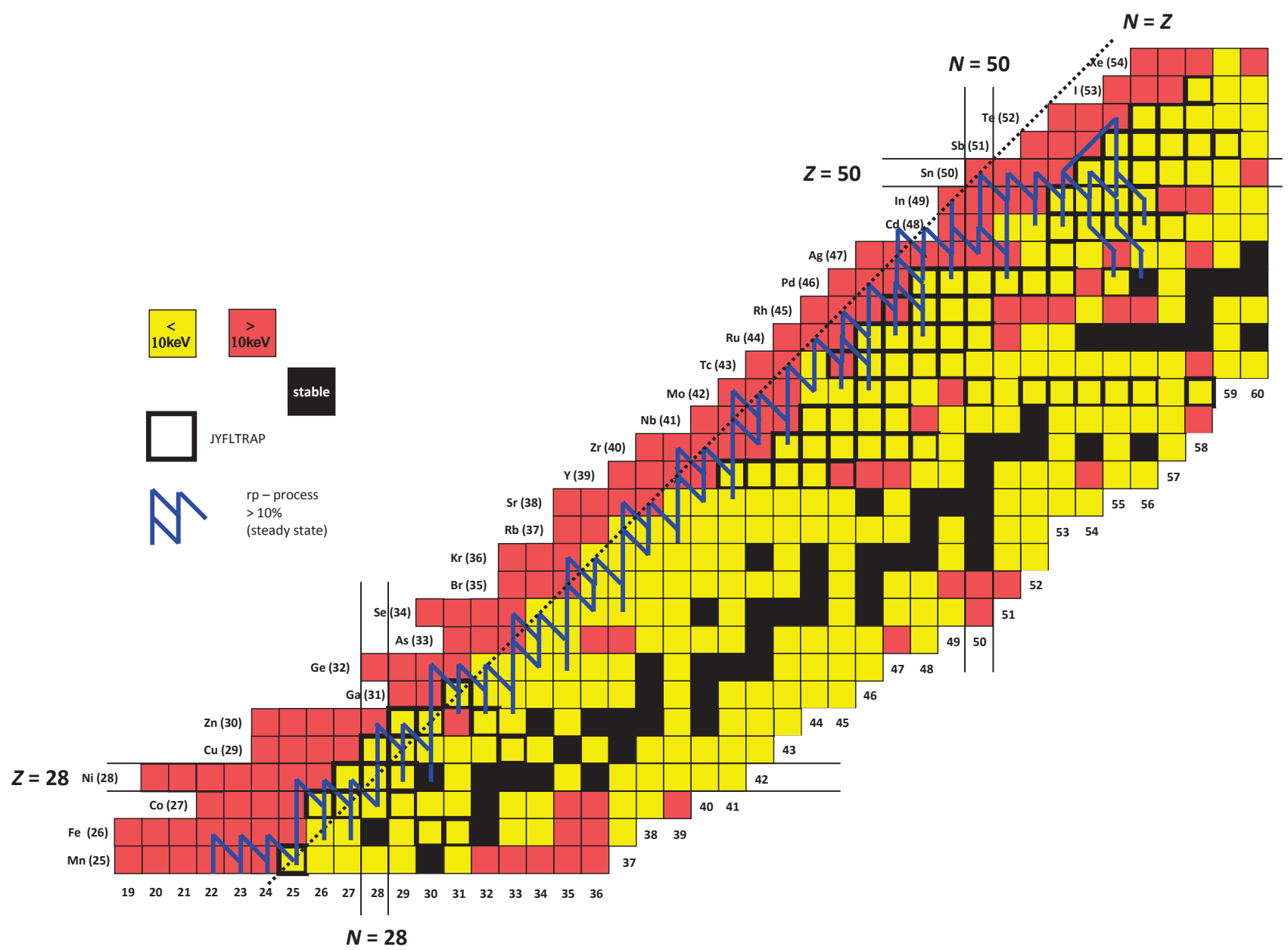

Fig. 1. (Color online) Chart of the nuclides showing the neutron-deficient nuclides measured at JYFLTRAP (bold black squares) and the nuclides whose mass excesses are known better than $10 \mathrm{keV}$ (in yellow) and worse than $10 \mathrm{keV}$ (in red, including the nuclides not yet experimentally determined). The main $r p$-process path (in blue) is plotted for conditions where the thermonuclear burning proceeds in steady-state.

2.1(15) keV. When our new mass value is combined with the proton separation energy of Ref. [56], a mass excess value, which is more precise than the previous measurements and agrees with the adopted AME03 value, is obtained for ${ }^{32} \mathrm{Cl}$. In Ref. [27], the quadratic IMME fit was performed with six different data sets corresponding to two different values of ${ }^{32} \mathrm{P}[52,57]$ and three different values of ${ }^{32} \mathrm{Si}[52,57-59]$. The overall result of the quadratic fits is that the IMME fails significantly $\left(\chi^{2} / n>6.5\right)$ in all data sets. The cubic term $d$ was found to depend strongly on the data set: the values varied from $d=0.51(15) \mathrm{keV}$ to $d=1.00(13) \mathrm{keV}$. In future, further mass measurements of ${ }^{32} \mathrm{Ar},{ }^{32} \mathrm{Cl},{ }^{32} \mathrm{P}$, and ${ }^{32} \mathrm{Si}$, should solve these discrepancies and really validate the breakdown of the IMME.

\subsection{Mass measurements around ${ }^{56} \mathrm{Ni}$}

Mass measurements around the doubly-magic $N=Z \mathrm{nu}-$ cleus ${ }^{56} \mathrm{Ni}$ provide essential data for the studies of e.g. isospin symmetry, mirror nuclei, Coulomb displacement energies, neutron-proton pairing, and shell-gap energies.
At JYFLTRAP, $Q_{\mathrm{EC}}$ values of the mirror nuclei ${ }^{53} \mathrm{Co}$, ${ }^{55} \mathrm{Ni},{ }^{57} \mathrm{Cu}$, and ${ }^{59} \mathrm{Zn}$, as well as of the $N=Z$ nuclei ${ }^{54} \mathrm{Co}$, ${ }^{56} \mathrm{Ni},{ }^{58} \mathrm{Cu},{ }^{60} \mathrm{Zn}$, and ${ }^{62} \mathrm{Ga}$ have been precisely measured (see Refs. [37-39]). In addition to $Q_{\mathrm{EC}}$ values, a few proton separation energies $\left(S_{\mathrm{p}}\right)$ have been directly measured by using the $(A-1, Z-1)$ nuclide as a reference for the $(A, Z)$ nuclide [38]. In order to obtain more accurate mass measurements, a network calculation of 17 frequency ratio measurements between 13 nuclides has been performed around ${ }^{56} \mathrm{Ni}[38]$. In this way, also the masses of reference nuclides, such as ${ }^{55} \mathrm{Co}$ and ${ }^{58} \mathrm{Ni}$, and ${ }^{59} \mathrm{Cu}$, could be evaluated. The obtained masses for ${ }^{55} \mathrm{Ni},{ }^{56} \mathrm{Ni}$, and ${ }^{57} \mathrm{Cu}$ agree well with the AME03 values [52], but are 15, 26, and 31 times more precise, respectively.

The most significant deviations (around $2 \sigma$ ) to the AME03 values in this mass region have been found for copper isotopes ${ }^{58} \mathrm{Cu}(-3.6(17) \mathrm{keV})$ and ${ }^{62} \mathrm{Cu}(10.6(42) \mathrm{keV})$. The measured JYFLTRAP mass of ${ }^{62} \mathrm{Cu}$ is higher than the adopted AME03 value, which was based on measurements using the ${ }^{62} \mathrm{Ni}(\mathrm{p}, \mathrm{n}){ }^{62} \mathrm{Cu}$ reaction $[60,61]$ and the $\beta$-decay energies of ${ }^{62} \mathrm{Cu}[62-64]$. The first two of the three $\beta$-decay 
experiments $[62,63]$ have underestimated the $\beta$-decay energy, which results in a too low adopted AME03 value.

The mass of ${ }^{58} \mathrm{Cu}$ has already been measured with the JYFLTRAP purification trap in 2004 [43]. The new JYFLTRAP mass value for ${ }^{58} \mathrm{Cu}$ agrees with the old purification trap measurement [43] but differs from the precise $(p, n)$ threshold energy measurements [65-67]. However, when the $(p, n)$ measurements are revised with the updated mass values [68] an agreement with JYFLTRAP is obtained.

Absolute deviations to AME03 are rather small in this mass region. The biggest deviations (in $\mathrm{keV}$ ) belong to ${ }^{59} \mathrm{Zn}(47(40) \mathrm{keV})$ and ${ }^{60} \mathrm{Zn}(15(11) \mathrm{keV})$. For those cases the $Q$ values derived from ${ }^{58} \mathrm{Ni}(\mathrm{p}, \pi)^{59} \mathrm{Zn}[69]$ and ${ }^{58} \mathrm{Ni}\left({ }^{3} \mathrm{He}, \mathrm{n}\right){ }^{60} \mathrm{Zn}[70]$ lead to slightly underestimated masses for ${ }^{59} \mathrm{Zn}$ and ${ }^{60} \mathrm{Zn}$. In addition to these nuclides, a difference was found for the proton-decaying high-spin isomer in ${ }^{53} \mathrm{Co}$ : the new JYFLTRAP value is $36(22) \mathrm{keV}$ lower than in AME03 [52].

$Q_{\mathrm{EC}}$ values of several $T=1 / 2$ mirror nuclei have been measured at JYFLTRAP. These experiments have been motivated by recent studies $[71,72]$ where corrected $f t$ values have been calculated for $T=1 / 2$ mirror transitions and the Conserved Vector Current Hypothesis has been tested. The $Q_{\mathrm{EC}}$ values of the $T=1 / 2$ nuclei ${ }^{31} \mathrm{~S}$, ${ }^{53} \mathrm{Co},{ }^{55} \mathrm{Ni},{ }^{57} \mathrm{Cu}$, and ${ }^{59} \mathrm{Zn}$ have been measured with a precision of better than $0.7 \mathrm{keV}$ at JYFLTRAP $[27,38]$. In addition, the $Q_{\mathrm{EC}}$ value of ${ }^{53} \mathrm{Co}^{\mathrm{m}}[38]$ has been measured with a precision of $1.2 \mathrm{keV}$. The largest differences to the AME03 values have been found at ${ }^{53} \mathrm{Co}^{\mathrm{m}}$ and ${ }^{59} \mathrm{Zn}$. The $Q_{\mathrm{EC}}$ values of ${ }^{45} \mathrm{~V}$ and ${ }^{49} \mathrm{Mn}$ have been measured in 2010 but the results have not yet been published. In the future these measurements will be continued with ${ }^{47} \mathrm{Cr}$ and ${ }^{51} \mathrm{Fe}$.

In addition to the $T=1 / 2$ mirror nuclei, the $Q_{\mathrm{EC}}$ values of the $T=1$ nuclei ${ }^{56} \mathrm{Ni},{ }^{58} \mathrm{Cu}$, and ${ }^{60} \mathrm{Zn}$ have been measured at JYFLTRAP. Interestingly, the JYFLTRAP $Q_{\mathrm{EC}}$ value of ${ }^{58} \mathrm{Cu}$ was found to be $4.6(15) \mathrm{keV}$ lower than in AME03. This $Q_{\mathrm{EC}}$ value has to be taken into account in studies of isospin symmetry among $A=58$ nuclei [73].

There have been no mass measurements for the $r p$ process between ${ }^{62} \mathrm{Ga}$ and ${ }^{80} \mathrm{Y}$ at JYFLTRAP. This region has been quite well covered by other Penning traps. At ISOLTRAP, Ga, Se, $\mathrm{Br}, \mathrm{Kr}, \mathrm{Rb}$, and $\mathrm{Sr}$ isotopes have been extensively studied [74-79]. Ga, Ge, As, Se, and $\mathrm{Br}$ isotopes relevant for the $r p$-process have been determined with LEBIT $[18,80]$, and Se, As, and Ge isotopes at $A=$ 68 with CPT [81].

\subsection{Mass measurements around $A=80-100$}

Neutron-deficient nuclei filling up neutron and proton $g_{9 / 2}$ shells in the mass region $A=80-100$ provide an abundant region for nuclear structure studies. Strong protonneutron interaction and competition between single-particle and collective motions result in the presence of many isomers and collective features. Spin-gap isomers, such as in ${ }^{94} \mathrm{Ag}[82-84]$ or ${ }^{95} \mathrm{Pd}[85]$, or shape isomers like in ${ }^{72} \mathrm{Kr}$

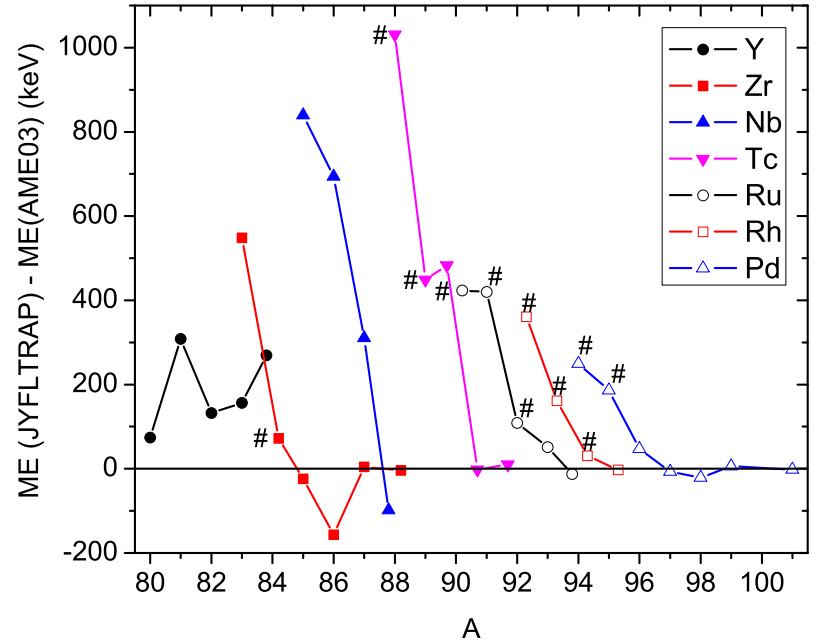

Fig. 2. (Color online) JYFLTRAP mass excess values compared to the AME03 values for the measured $\mathrm{Y}, \mathrm{Zr}, \mathrm{Nb}, \mathrm{Tc}$, $\mathrm{Ru}, \mathrm{Rh}$ and Pd isotopes. '\#' denotes that the AME03 value has been based on extrapolations. No error bars have been included in order to show more clearly the trend of the differences.

[86] have gained a lot of attention. Shell-model calculations are possible for these nuclides: in many cases it is sufficient to include only the $1 p_{1 / 2}$ and $0 g_{9 / 2}$ shells. Experimental data are needed to test and develop shell model calculations and more sophisticated model spaces, such as $\left(1 p_{3 / 2}, 0 f_{5 / 2}, 1 p_{1 / 2}, 0 g_{9 / 2}\right)$ [84] or $\left(0 g_{9 / 2}, 1 d_{5 / 2}, 0 g_{7 / 2}\right.$, $\left.1 d_{3 / 2}, 2 s_{1 / 2}\right)[83]$.

The mass measurements conducted at JYFLTRAP in this mass region have revealed large deviations to the AME03 values. Many of the measured mass excess values, for example ${ }^{83,85} \mathrm{Zr}$ and ${ }^{85-88} \mathrm{Nb}$, have earlier been based on $\beta$-decay endpoint energies, which have a tendency to underestimate the masses due to unobserved feeding to higher-lying states in the daughter nuclides (pandemonium effect [87]). This problem is likely to play a stronger role in nuclides further from stability. Thus, the more exotic the nuclide, the more it typically deviates from the JYFLTRAP value (see Fig. 2). The yttrium isotopes do not follow this trend since the AME03 value for ${ }^{80} \mathrm{Y}$ is based on a direct time-of-flight mass measurement at the ISN SARA cyclotron [88]. The mass of ${ }^{86} \mathrm{Zr}$ in AME03 is based on ${ }^{90} \mathrm{Zr}\left(\alpha,{ }^{8} \mathrm{He}\right)^{86} \mathrm{Zr}$ reactions [89] and not on $\beta$ decay energies. Many nuclides $-{ }^{84} \mathrm{Zr},{ }^{88,89} \mathrm{Tc},{ }^{90-92} \mathrm{Ru}$, ${ }^{92-94} \mathrm{Rh}$, and ${ }^{94-95} \mathrm{Pd}$ - have been measured for the first time at JYFLTRAP. As can be seen from Fig. 2, the extrapolated mass excess values of AME03 are typically too small. In other words, JYFLTRAP has found these nuclei to be less bound.

In the mass region below $Z=50$, the mass excesses of ${ }^{89-92} \mathrm{Tc}, \quad{ }^{90-92,94} \mathrm{Ru},{ }^{92-93} \mathrm{Rh}$ as well as ${ }^{99,101} \mathrm{Ag}$, ${ }^{101-104} \mathrm{Cd},{ }^{102-105}$ In have been measured with the SHIPTRAP Penning trap at GSI $[19,90]$. In general, SHIPTRAP and JYFLTRAP results agree well with each other (see Fig. 3). Small differences are found for ${ }^{92} \mathrm{Tc}\left(\Delta_{S-J}=\right.$ $15(12) \mathrm{keV})$ and ${ }^{90} \mathrm{Ru}\left(\Delta_{S-J}=17(11) \mathrm{keV}\right)$. Differences are also found for ${ }^{101,102,104} \mathrm{Cd}$, where ISOLTRAP data 
Table 2. Isomers taken into account in the determination of the ground-state masses in the $A \approx 80-100$ region.

\begin{tabular}{lll|lll}
\hline Isomer & $E_{\mathrm{x}}(\mathrm{keV})$ & Ref. & Isomer & $E_{\mathrm{x}}(\mathrm{keV})$ & Ref. \\
\hline${ }^{83} \mathrm{Y}^{\mathrm{m}}$ & $61.98(11)$ & {$[92]$} & ${ }^{88} \mathrm{Tc}^{\mathrm{m}}$ & $300 \#$ & {$[92]$} \\
${ }^{84} \mathrm{Y}^{\mathrm{m}}$ & 67 & {$[93]$} & ${ }^{92} \mathrm{Rh}^{\mathrm{m}}$ & $50 \#$ & {$[94,95]$} \\
${ }^{85} \mathrm{Nb}^{\mathrm{m}}$ & $\geq 69$ & {$[96]$} & ${ }^{100} \mathrm{Ag}^{\mathrm{m}}$ & $15.52(16)$ & {$[92]$} \\
${ }^{87} \mathrm{Nb}^{\mathrm{m}}$ & $3.84(14)$ & {$[92]$} & ${ }^{104} \mathrm{In}^{\mathrm{m}}$ & $93.48(10)$ & {$[92]$} \\
${ }^{88} \mathrm{Nb}^{\mathrm{m}}$ & $40(140)$ & {$[92]$} & & & \\
\hline
\end{tabular}

[17] are in agreement with JYFLTRAP, not with SHIPTRAP. Many of these masses have also been measured with CPT but the preliminary graphical data [91] for 90,91 Mo, $90-93 \mathrm{Tc}$ ${ }^{93-94} \mathrm{Ru},{ }^{94,95} \mathrm{Rh},{ }^{104-107} \mathrm{In},{ }^{104-108} \mathrm{Sn},{ }^{107-108} \mathrm{Sb}$ could not be included in this comparison ${ }^{1}$. The JYFLTRAP mass excess values used here have been updated with the new molybdenum masses measured at JYFLTRAP discussed in a separate article in this special issue of Hyperfine Interactions.

Many open questions concerning the neutron-deficient nuclides around $A=80-100$ remain. For example, whether an observed state is a pure ground state, an isomeric state, or a mixture of these should be investigated and verified for some of the measured nuclides. Corrections due to a possible mixture of ground and isomeric states or due to unknown level scheme have been applied according to Eq. (14) of Ref. [97] and have been added quadratically to the experimental uncertainties for the nuclei listed in Table 2. However, in some cases isomers might not be known, or information is uncertain. For example, no correction has been applied to ${ }^{86} \mathrm{Nb}\left(E_{\mathrm{x}}=250(160) \# \mathrm{keV}\right.$ [92]) since this isomer is considered as uncertain. Clearly more experiments are needed to clarify the low-lying level structure of nuclides in this mass region, and revisions of some mass values might become necessary as a consequence.

\subsection{Mass measurements above ${ }^{100} \mathrm{Sn}$}

Masses of ${ }^{104-108} \mathrm{Sn},{ }^{106-110} \mathrm{Sb},{ }^{108,109} \mathrm{Te}$, and ${ }^{111} \mathrm{I}$ in the endpoint region of the $r p$-process have been measured with JYFLTRAP [3]. The masses of $106,108,110 \mathrm{Sb}$ have been experimentally determined for the first time, and found to agree with the AME03 extrapolations. Overall agreement of the JYFLTRAP mass values with the AME03 values in this region is fairly good: the only deviations occur at ${ }^{104,106,108} \mathrm{Sn}$, and ${ }^{109} \mathrm{Te}$. In the following, JYFLTRAP results are compared to previous experiments nuclide by nuclide (see also Figs. 4, 5, and 6).

${ }^{104} \mathrm{Sn}$ : The mass of ${ }^{104} \mathrm{Sn}$ has been previously measured via $\beta$-decay endpoint energies $[98,99]$. It can also be determined from the $\alpha$-decay energy of ${ }^{108} \mathrm{Te}$ [102-105]. The $\beta$-decay results and the $Q_{\alpha}$-values from Refs. [103,

1 The results from J. Fallis et al., Phys. Rev. C (2011) on Nb, $\mathrm{Mo}, \mathrm{Tc}, \mathrm{Ru}$, and $\mathrm{Rh}$ isotopes were published at proof stage, and thus could not be included here.

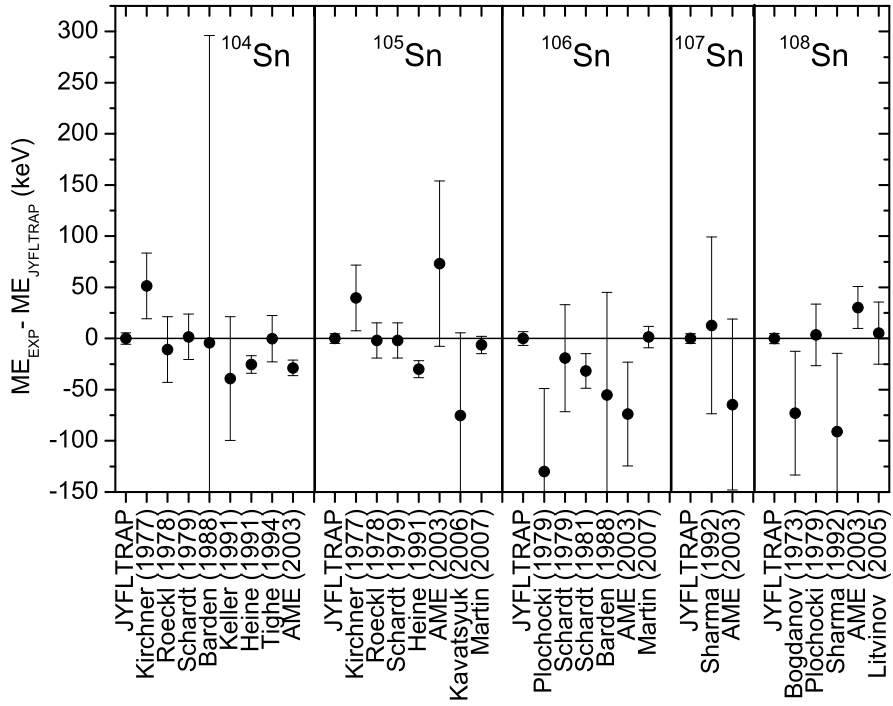

Fig. 4. JYFLTRAP mass excess values for Sn isotopes compared to other experiments. Beta [98-101], alpha [102-106], proton [107] and $\beta$-delayed proton decay [108] energies have been used with the latest mass values obtained at JYFLTRAP whenever possible. Direct mass measurements performed at SHIPTRAP [90] for ${ }^{105,106} \mathrm{Sn}$, and at ESR for ${ }^{108} \mathrm{Sn}$ [109] as well as the values based on the mass ratios of ${ }^{107} \mathrm{Sn} /{ }^{106} \mathrm{Sn}$ and ${ }^{107} \mathrm{Sn} /{ }^{108} \mathrm{Sn}[110]$ are also shown in the figure. The AME03 values [52] deviate from the JYFLTRAP values of ${ }^{104,106,108} \mathrm{Sn}$.

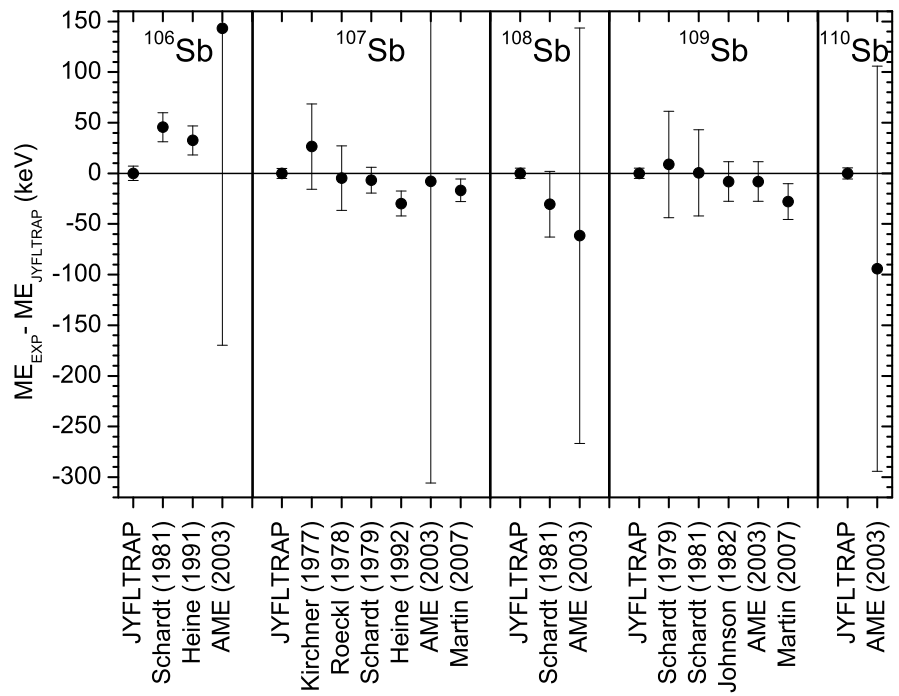

Fig. 5. JYFLTRAP mass excess values for $\mathrm{Sb}$ isotopes compared to other experiments. Beta [111] and alpha decay [102104,106,112] energies have been combined with the latest JYFLTRAP values in the calculations. SHIPTRAP mass values for ${ }^{107,109} \mathrm{Sb}$ [90] deviate from the new JYFLTRAP values. The AME03 [52] mass values of ${ }^{106-108,110} \mathrm{Sb}$ are based only on extrapolations. The JYFLTRAP values agree with these AME03 values. 


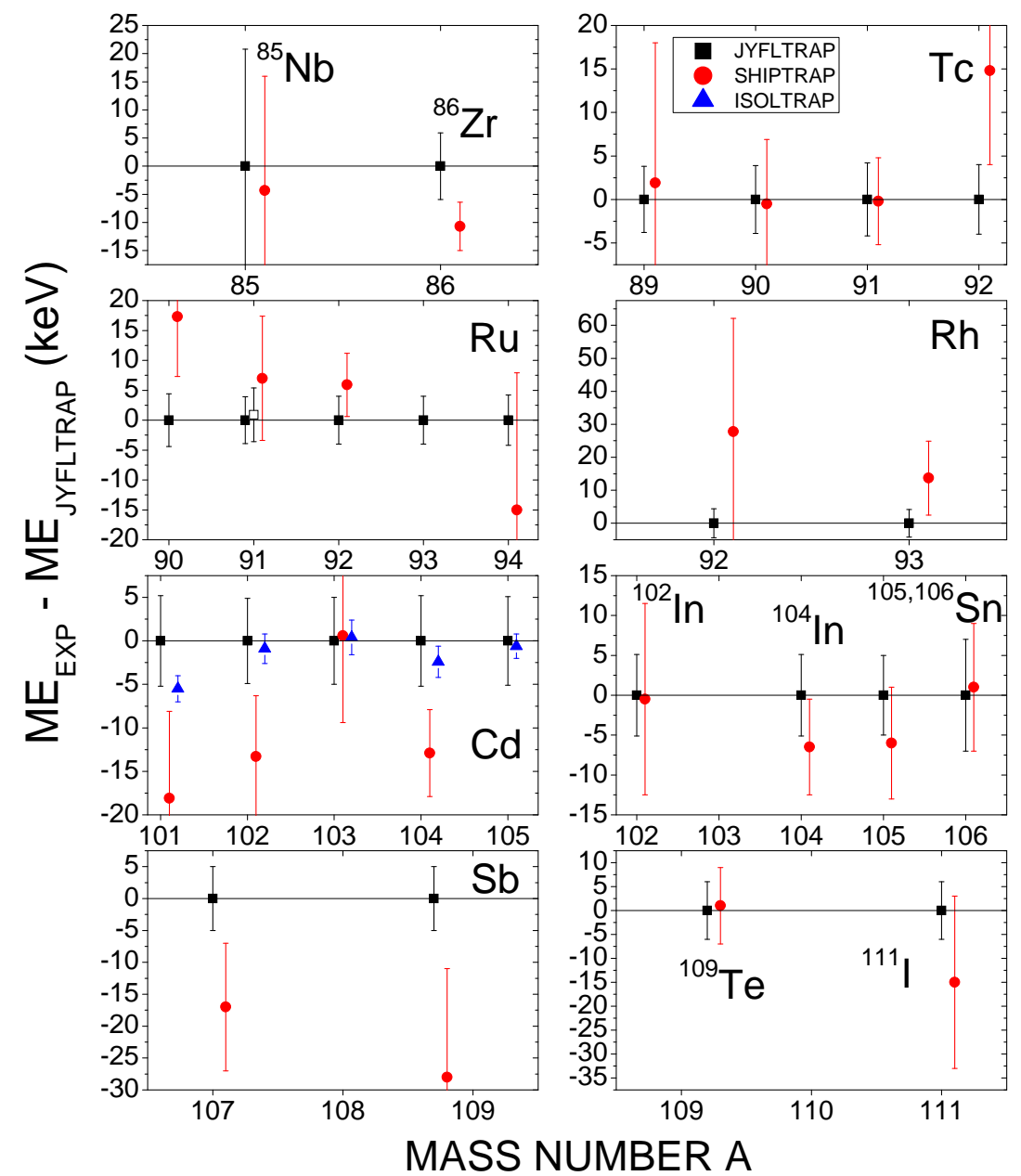

Fig. 3. (Color online) Differences between the JYFLTRAP mass excess values (black squares) and the values obtained at SHIPTRAP (red circles) [19,90] and ISOLTRAP (blue triangles) [17]. The error bars represent the original experimental uncertainties and not the uncertainties of the differences. The isomer or unknown level scheme corrections have not been applied for ${ }^{92} \mathrm{Rh}$ and ${ }^{104} \mathrm{In}$ in this figure. ${ }^{91} \mathrm{Ru}$ has been measured against ${ }^{85} \mathrm{Rb}$ (solid square) and ${ }^{94} \mathrm{Mo}$ (open square) at JYFLTRAP. The masses measured against Mo isotopes at JYFLTRAP have been corrected with the new molybdenum mass values from JYFLTRAP (for details, see a separate article in this special issue of Hyperfine Interactions).

104] agree with the JYFLTRAP result but the $\alpha$-decay energies from Refs. [102,105] differ from it. The AME03 value, which is mainly based on the $\alpha$-decay energies, is significantly lower than the JYFLTRAP value.

${ }^{105} \mathrm{Sn}$ : The mass of ${ }^{105} \mathrm{Sn}$ can be determined from the $Q_{\alpha}$-values of ${ }^{109} \mathrm{Te}[102-105]$. The $Q_{\alpha}$-values from Refs. [103,104] agree with the JYFLTRAP result but as in the case of ${ }^{104} \mathrm{Sn}$ the values from Refs. [102,105] differ: $Q_{\alpha}$ from Ref. [102] is around $40 \mathrm{keV}$ smaller and the value from Ref. [105] is around $30 \mathrm{keV}$ higher than the $Q_{\alpha}$-value obtained with JYFLTRAP. The JYFLTRAP result agrees with the $\beta$-endpoint measurement of ${ }^{105} \mathrm{Sn}[100]$ and the SHIPTRAP value [90].

${ }^{106} \mathrm{Sn}$ : The $Q_{\mathrm{EC}}$ value of ${ }^{106} \mathrm{Sn}$ from Ref. [98], the $Q_{\alpha}$ value of ${ }^{110}$ Te from Ref. [104] and the SHIPTRAP value [90] agree well with the result obtained at JYFLTRAP. However, the values based on the $Q_{\mathrm{EC}}$ value from Ref. [101] and the $Q_{\alpha}$-value of ${ }^{110}$ Te from Ref. [106] differ slightly from the JYFLTRAP mass excess value for ${ }^{106} \mathrm{Sn}$. As a result, the AME03 value differs from the JYFLTRAP value.

${ }^{107} \mathrm{Sn}$ : The mass of ${ }^{107} \mathrm{Sn}$ has earlier been based on the mass ratio measurement of ${ }^{107} \mathrm{Sn}$ to ${ }^{106} \mathrm{Sn}$ [110]. The mass value determined at JYFLTRAP agrees well with it.

${ }^{108} \mathrm{Sn}$ : The $\beta$-delayed proton-decay of ${ }^{109} \mathrm{Te}[108]$, the mass ratio of ${ }^{108} \mathrm{Sn}$ to ${ }^{107} \mathrm{Sn}[110]$, and the AME03 value [52] disagree slightly from the JYFLTRAP value. The result from the $\beta$-decay of ${ }^{108} \mathrm{Sn}$ [101] and the direct mass measurement performed at ESR [109] agree very well with JYFLTRAP. The differences between the JYFLTRAP and the AME03 mass excess values for ${ }^{109} \mathrm{Te}$ and ${ }^{107} \mathrm{Sn}$ explain why the mass excess values for ${ }^{108} \mathrm{Sn}$ derived from Refs. $[108,110]$ differ from the AME03 value (see Fig. 4).

${ }^{106} \mathrm{Sb}$ : The mass of ${ }^{106} \mathrm{Sb}$ has been measured for the first time with JYFLTRAP and the result agrees with the extrapolation based on systematic trends in AME03 [52].

${ }^{107} \mathrm{Sb}$ : The mass of ${ }^{107} \mathrm{Sb}$ has been directly measured at SHIPTRAP [90]. The value differs from the JYFLTRAP 


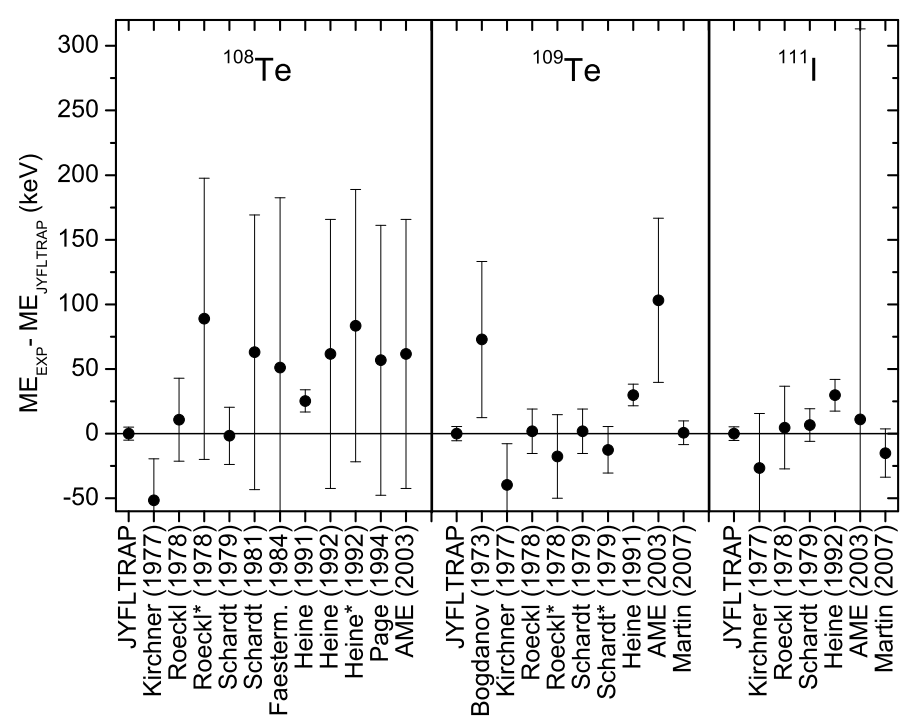

Fig. 6. JYFLTRAP mass excess values for Te and I isotopes compared to other experiments. Alpha [102-106,112,113], proton $[112,114]$ and $\beta$-delayed proton decay [108] energies have been used with the latest JYFLTRAP values in the calculations of the mass values. The SHIPTRAP measurements of ${ }^{109} \mathrm{Te}$ and ${ }^{111} \mathrm{I}[90]$ agree well with the JYFLTRAP data. The AME03 value [52] for ${ }^{109}$ Te deviates from the JYFLTRAP data.

value by $17(12) \mathrm{keV}$. The mass can also be determined from the $\alpha$-decay energies of ${ }^{111} \mathrm{I}[102-104,112]$ and the mass of ${ }^{111} \mathrm{I}[3]$. All $\alpha$-decay results except the one from Ref. [112] agree with JYFLTRAP.

${ }^{108} \mathrm{Sb}$ : The mass of ${ }^{108} \mathrm{Sb}$ has been experimentally determined at JYFLTRAP for the first time. The obtained value agrees with the extrapolated value [52]. The mass can also be calculated from the $Q_{\alpha}$ of ${ }^{112} \mathrm{I}[106]$ and the mass of ${ }^{112} \mathrm{I}$ measured at SHIPTRAP [90]. The value agrees with the JYFLTRAP result.

${ }^{109} \mathrm{Sb}$ : The mass of ${ }^{109} \mathrm{Sb}$ has been measured at SHIPTRAP. The JYFLTRAP value differs from it by $28(18) \mathrm{keV}$. The $\beta$-endpoint energy of ${ }^{109} \mathrm{Sb}[111]$ and the $\alpha$-decay energies of ${ }^{113}$ I $[104,106]$ together with the mass of ${ }^{113}$ I measured at SHIPTRAP [90] agree with JYFLTRAP

${ }^{110} \mathrm{Sb}$ : The mass of ${ }^{110} \mathrm{Sb}$ has been measured for the first time at JYFLTRAP. The mass excess value agrees with the extrapolated value of AME03 [52].

${ }^{108}$ Te: The mass of ${ }^{108}$ Te can be determined in several ways. Firstly, the experimental $Q_{\alpha}$-values of ${ }^{108} \mathrm{Te}[102-$ $105]$ and the mass excess of ${ }^{104} \mathrm{Sn}$ yield similar agreements and disagreements as for ${ }^{104} \mathrm{Sn}$ for which the mass excess values were derived from these same $Q_{\alpha}$ values and the mass excess of ${ }^{108} \mathrm{Te}$. Secondly, the $Q_{\alpha}$-values of ${ }^{112} \mathrm{Xe}$ $[103,106,112,113]$ and the AME03 value for ${ }^{112}$ Xe can be used to estimate the mass of ${ }^{108} \mathrm{Te}$. These values agree with the JYFLTRAP result. Thirdly, the proton decay data of ${ }^{109} \mathrm{I}[112,114]$ combined with the AME03 value for ${ }^{109} \mathrm{I}$ agree with the JYFLTRAP measurement.

${ }^{109}$ Te: The mass of ${ }^{109} \mathrm{Te}$ has previously been measured at SHIPTRAP [90] and the values agree with the JYFLTRAP results. It can also be derived from the $Q_{\alpha^{-}}$ values of ${ }^{109} \mathrm{Te}[102-105]$ with the ${ }^{105} \mathrm{Sn}$ mass excess value, $Q_{\alpha}$-values of ${ }^{113} \mathrm{Xe}[103,104]$ with the ${ }^{113} \mathrm{Xe}$ mass measured at SHIPTRAP, and from the $\beta$-delayed proton data of ${ }^{109} \mathrm{Te}[108]$ and the mass of ${ }^{108} \mathrm{Sn}$. Of these results, only Refs. $[103,104]$ agree with JYFLTRAP. The AME03 value [52] based mainly on Ref. [108] is significantly higher than the JYFLTRAP mass excess value.

${ }^{111} \mathbf{I}$ : The mass of ${ }^{111} \mathrm{I}$ has also been measured at SHIPTRAP [90] and it agrees with JYFLTRAP. It can also be determined from the $Q_{\alpha}$-values of ${ }^{111} \mathrm{I}[102-104,112]$ with the mass of ${ }^{107} \mathrm{Sb}$. The measurements agree with the present data except for the result from Ref. [112].

\subsection{Energy systematics: $S_{\mathbf{p}}, Q_{\alpha}$, and $S_{2 \mathbf{p}}$ values}

Proton separation energies $\left(S_{\mathrm{p}}\right)$ are important for the calculations of proton-capture rates and for the modeling of the astrophysical $r p$ - or $\nu p$-processes, since the proton capture rates and obtained abundances depend exponentially on the $S_{\mathrm{p}}$ values. At JYFLTRAP, the mass excesses for both the $(A, Z)$ and $(A-1, Z-1)$ nuclides, and thus the $S_{\mathrm{p}}$ values, have been determined for 41 nuclides. The measured masses have an impact on altogether $138 S_{\mathrm{p}}$ values. For ${ }^{56} \mathrm{Ni},{ }^{57} \mathrm{Cu},{ }^{59} \mathrm{Zn}$, and ${ }^{60} \mathrm{Zn}$, a sub-keV precision in the proton separation energy has been achieved by using the $(A-1, Z-1)$ nuclide as a reference in the mass measurement. Figure 7 shows a comparison of the $S_{\mathrm{p}}$ values calculated entirely from JYFLTRAP masses with the values of AME03.

The biggest deviations in the proton separation energies compared to the AME03 values are found for $\mathrm{Nb}$, Tc, Zr and Mo isotopes (see Table 3). In addition, the $S_{\mathrm{p}}$ values of ${ }^{58} \mathrm{Cu},{ }^{59} \mathrm{Zn},{ }^{60} \mathrm{Zn},{ }^{88} \mathrm{Nb},{ }^{104} \mathrm{In}$, and ${ }^{109} \mathrm{Sb}$ differ slightly from AME03 (see Fig. 7). The deviations to the AME03 values are $-3.5(26), 50(40), 15(11),-100(100)$, $-110(90)$, and $40(30) \mathrm{keV}$, respectively. For the proton separation energies, for which either the mass of the $(A, Z)$ or $(A-1, Z-1)$ nuclide has been determined at JYFLTRAP, the values for ${ }^{82} \mathrm{Zr},{ }^{84} \mathrm{Nb},{ }^{89} \mathrm{Ru},{ }^{91} \mathrm{Rh},{ }^{93} \mathrm{Pd}$, and ${ }^{95} \mathrm{Ag}$ are partly based on extrapolated AME03 values, and thus, new experimental data may change those values dramatically. In addition, the $S_{\mathrm{p}}$ values for ${ }^{62} \mathrm{Cu},{ }^{63} \mathrm{Zn}$, ${ }^{82-83} \mathrm{Y},{ }^{86} \mathrm{Zr}$, and ${ }^{106,108} \mathrm{Sn}$ disagree with the AME03 values. In the lighter mass region, the $S_{\mathrm{p}}$ values for ${ }^{23,26} \mathrm{Al}$, ${ }^{26-27} \mathrm{Si},{ }^{31} \mathrm{~S},{ }^{34} \mathrm{Cl},{ }^{42} \mathrm{Ti}$, and ${ }^{46} \mathrm{~V}$ differ from the AME03 values.

In the SnSbTe region, $\alpha$ separation energies become sufficiently small for $\alpha$ decay to become energetically possible, and for $(\mathrm{p}, \alpha)$ and $(\gamma, \alpha)$ reactions to play an important role in astrophysical environments. $Q_{\alpha}$ values are therefore needed. The $Q_{\alpha}$ values of ${ }^{108,109} \mathrm{Te}$ and ${ }^{111} \mathrm{I}$ have been precisely determined at JYFLTRAP by measuring the masses of the corresponding mother and daughter nuclides (see Table 4). The JYFLTRAP $Q_{\alpha}$ value for ${ }^{108} \mathrm{Te}$ is significantly lower than in AME03. By combining the results of JYFLTRAP [3,41] with SHIPTRAP [90], also the $Q_{\alpha}$ values for ${ }^{105-108} \mathrm{Sn}$ and ${ }^{106-109} \mathrm{Sb},{ }^{110-112} \mathrm{Te}$, ${ }^{112-113} \mathrm{I}$, and ${ }^{113} \mathrm{Xe}$ have been obtained. For these $Q_{\alpha}$ val- 

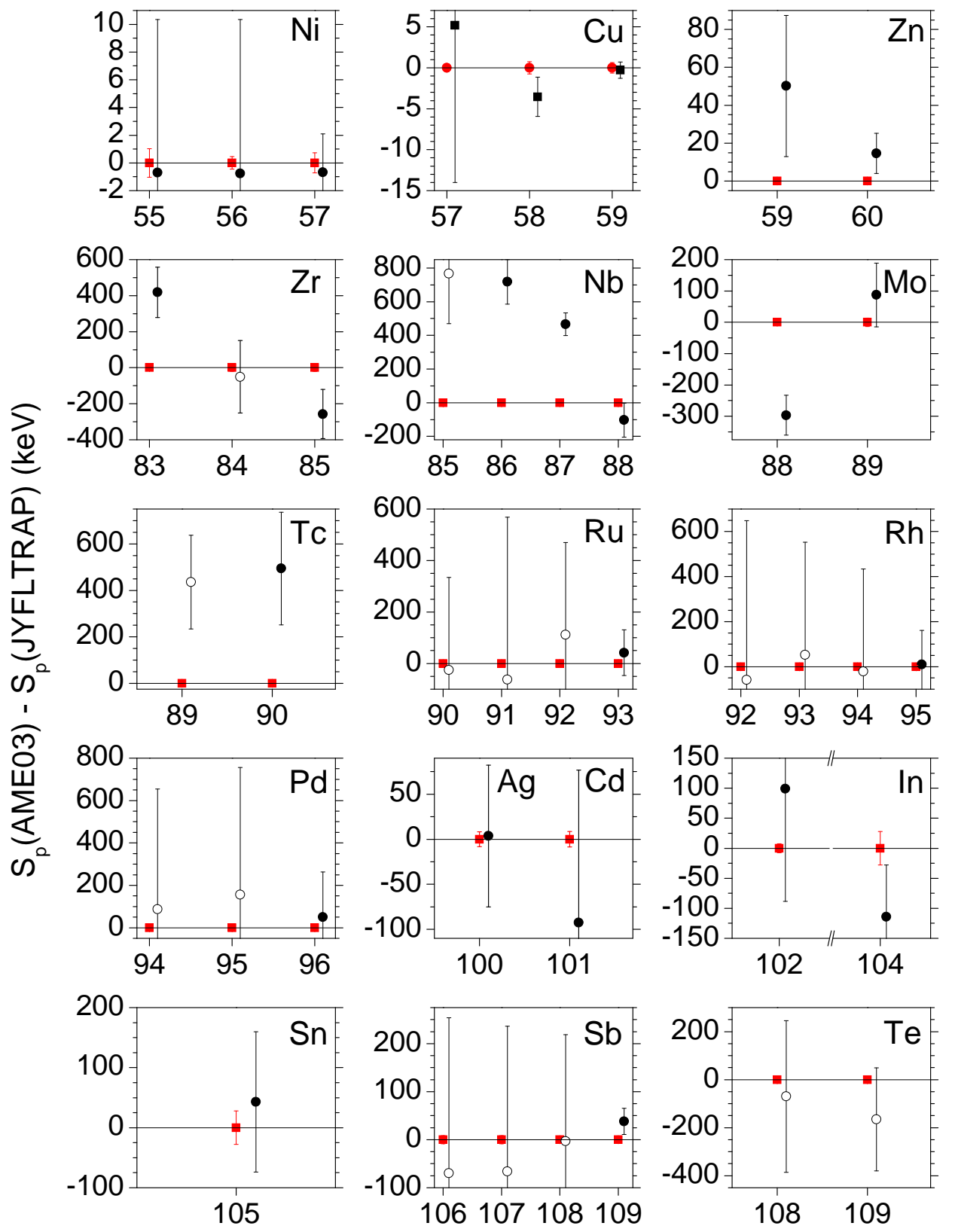

\section{MASS NUMBER A}

Fig. 7. (Color online) A comparison of proton separation energies measured at JYFLTRAP (masses both for $(A, Z)$ and $(A-1, Z-1)$ measured) to the values from AME03 [52]. The JYFLTRAP values are shown as red squares, the AME03 values as black circles and the extrapolated values in AME03 by open black circles. The uncertainty of the JYFLTRAP $S_{\mathrm{p}}$ value is in many cases much smaller than the size of the red square.

ues, deviations to AME03 are found for ${ }^{108} \mathrm{Sn},{ }^{109} \mathrm{Sb}$, and $110-112 \mathrm{Te}$.

Two-proton separation energies $S_{2 p}$ plotted against the proton number $Z$ often show continuous and smooth behavior over several isotopes. Deviations from the trend reflect possible subshell or shell closures or an onset of deformation. The shell closure at $Z=50$ is seen in Fig. 8, where two-proton separation energies obtained at JYFLTRAP (combined with the Penning trap data from Refs. $[17,90])$ are plotted against proton number $Z$ : the energies fall down steeply at $Z=50$. The trend is very similar to the AME03 values for the isotonic chains above $N=47$. Below $N=47$, the isotonic chains lie at lower energies than in AME03, since the mass excess values of $\mathrm{Nb}, \mathrm{Mo}$ and Tc isotopes have been too low in AME03. In addition, these lines are steeper when proceeding towards more proton-rich nuclides. The new Penning trap measurements have shown that these nuclides are less proton bound than in AME03. 
Table 3. Largest observed differences (in $\mathrm{keV}$ ) to the AME03 proton separation energies at JYFLTRAP. For the nuclides marked with ${ }^{a}$, the $Z-1$ nuclide has not been measured at JYFLTRAP and AME03 has been adopted. The mass excesses for the nuclides marked with ${ }^{b}$ have been adopted from AME03 but the corresponding $Z-1$ nuclides have been measured with JYFLTRAP. '\#' denotes that the used AME03 value is based on extrapolations. The big deviations for ${ }^{86,87} \mathrm{Mo}$ and ${ }^{88} \mathrm{Tc}$ vanish when the new mass values from Ref. [115] are adopted.

\begin{tabular}{|c|c|c|c|}
\hline Nuclide & $\begin{array}{l}S_{\mathrm{p}} \\
(\mathrm{keV})\end{array}$ & $\begin{array}{l}S_{\mathrm{p}, \text { lit. }}[52] \\
(\mathrm{keV})\end{array}$ & $\begin{array}{l}S_{\mathrm{p}}-S_{\mathrm{p}, \mathrm{lit}} \\
(\mathrm{keV})\end{array}$ \\
\hline${ }^{81} \mathrm{Y}^{a}$ & $2692(9)$ & $3000(60)$ & $-310(60)$ \\
\hline${ }^{84} \mathrm{Y}^{a}$ & $4418(23)$ & $4650(90)$ & $-230(100)$ \\
\hline${ }^{82} \mathrm{Zr}^{b}$ & $5770(230) \#$ & $5460(230) \#$ & $310(320) \#$ \\
\hline${ }^{83} \mathrm{Zr}$ & $5137(9)$ & $5560(140)$ & $-420(140)$ \\
\hline${ }^{85} \mathrm{Zr}$ & $6538(21)$ & $6280(140)$ & $260(140)$ \\
\hline${ }^{84} \mathrm{Nb}^{b}$ & $3260(300) \#$ & 2710(310)\# & $550(430) \#$ \\
\hline${ }^{85} \mathrm{Nb}$ & $2178(22)$ & 2950(300)\# & $-770(300) \#$ \\
\hline${ }^{86} \mathrm{Nb}$ & $3248(8)$ & $3970(130)$ & $-720(130)$ \\
\hline${ }^{87} \mathrm{Nb}$ & $3201(9)$ & $3670(70)$ & $-470(70)$ \\
\hline${ }^{86} \mathrm{Mo}^{b}$ & $5540(440)$ & $4700(490)$ & $840(660)$ \\
\hline${ }^{87} \mathrm{Mo}^{b}$ & $5850(220)$ & $5160(240)$ & $690(330)$ \\
\hline${ }^{88} \mathrm{Mo}$ & $6102(8)$ & $5810(60)$ & $300(60)$ \\
\hline${ }^{88} \mathrm{Tc}^{a}$ & $1270(240)$ & $2300(300) \#$ & $-1030(380) \#$ \\
\hline${ }^{89} \mathrm{Tc}$ & $1997(6)$ & $2430(200) \#$ & $-440(200) \#$ \\
\hline${ }^{90} \mathrm{Tc}$ & $2998(6)$ & $3490(240)$ & $-500(240)$ \\
\hline${ }^{89} \mathrm{Ru}^{b}$ & $5120(510) \#$ & 4090(540)\# & 1030(740)\# \\
\hline${ }^{91} \mathrm{Rh}^{b}$ & $1510(400) \#$ & 1090(500)\# & $420(640) \#$ \\
\hline${ }^{93} \mathrm{Pd}^{b}$ & 3990(400)\# & $3630(570) \#$ & $360(700) \#$ \\
\hline${ }^{95} \mathrm{Ag}^{b}$ & 1290(400)\# & 1040(570)\# & $250(700) \#$ \\
\hline
\end{tabular}

Table 4. $Q_{\alpha}$ values from JYFLTRAP mass measurements [3, 41] combined with the SHIPTRAP results [90], and comparison to the literature values [52]. Only alpha decays for which both the mother and daughter have been measured at JYFLTRAP and/or SHIPTRAP and at least either of them has been measured at JYFLTRAP have been taken into account.

\begin{tabular}{llll}
\hline Nuclide & $\begin{array}{l}Q_{\alpha} \\
(\mathrm{keV})\end{array}$ & $\begin{array}{l}Q_{\alpha, l i t} \\
(\mathrm{keV})\end{array}$ & $\begin{array}{l}Q_{\alpha}-Q_{\alpha, l i t} \\
(\mathrm{keV})\end{array}$ \\
\hline${ }^{105} \mathrm{Sn}$ & $74(5)$ & $60(170)$ & $14(170)$ \\
${ }^{106} \mathrm{Sn}$ & $-116(6)$ & $-170(60)$ & $54(60)$ \\
${ }^{107} \mathrm{Sn}$ & $-286(6)$ & $-350(80)$ & $62(90)$ \\
${ }^{108} \mathrm{Sn}$ & $-527(6)$ & $-491(22)$ & $-36(23)$ \\
${ }^{106} \mathrm{Sb}$ & $1795(9)$ & $1950(330) \#$ & $-155(340)$ \\
${ }^{107} \mathrm{Sb}$ & $1558(10)$ & $1520(300) \#$ & $38(300)$ \\
${ }^{108} \mathrm{Sb}$ & $1309(7)$ & $1170(220) \#$ & $139(220)$ \\
${ }^{109} \mathrm{Sb}$ & $963(11)$ & $797(26)$ & $166(28)$ \\
${ }^{108} \mathrm{Te}$ & $3416(8)$ & $3445(4)$ & $-29(9)$ \\
${ }^{109} \mathrm{Te}$ & $3198(6)$ & $3230(50)$ & $-32(50)$ \\
${ }^{110} \mathrm{Te}$ & $2696(9)$ & $2723(16)$ & $-27(19)$ \\
${ }^{111} \mathrm{Te}$ & $2500(8)$ & $2670(110)$ & $-170(110)$ \\
${ }^{112} \mathrm{Te}$ & $2079(10)$ & $2310(170)$ & $-231(170)$ \\
${ }^{111} \mathrm{I}$ & $3265(7)$ & $3280(50)$ & $-15(50)$ \\
${ }^{112} \mathrm{I}$ & $2956(10)$ & $2990(50)$ & $-34(50)$ \\
${ }^{113} \mathrm{I}$ & $2708(10)$ & $2710(50)$ & $-2(50)$ \\
${ }^{113} \mathrm{Xe}$ & $3085(9)$ & $3090(50)$ & $-5(50)$ \\
\hline
\end{tabular}

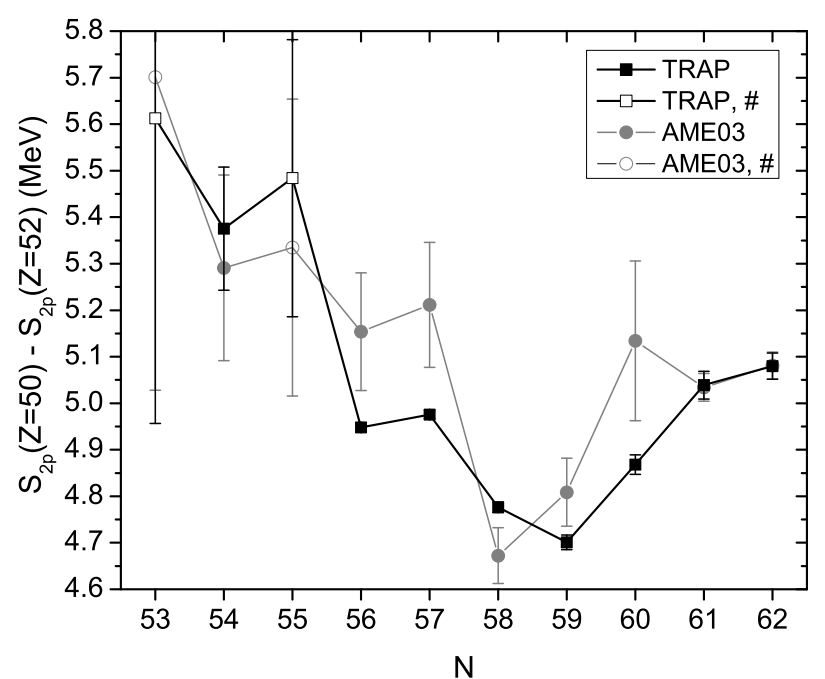

Fig. 9. Proton shell-gap energies at $Z=50$ determined from two-proton separation energies. Recent Penning trap data from JYFLTRAP [3, 41], SHIPTRAP [90], and ISOLTRAP [17] have been adopted whenever possible. The 'TRAP' data cover the values where at least one data set comes from a Penning-trap measurement and the missing data have been adopted from AME03. The 'AME03' data points are based only on AME03. '\#' marks that the used AME03 value is based on extrapolations.

After the JYFLTRAP masses for ${ }^{83} \mathrm{Zr}$ and ${ }^{85} \mathrm{Nb}$ had been determined, the $S_{2 \mathrm{p}}$ for ${ }^{85} \mathrm{Mo}(Z=42, N=43$, blue symbol) and ${ }^{87} \mathrm{Tc}(Z=43, N=44$, red symbol $)$ obtained with the AME03 masses for ${ }^{85} \mathrm{Mo}$ and ${ }^{87} \mathrm{Tc}$ show an extreme deviation from the systematic trend in their respective isotonic chains (blue line for $N=43$ and red line for $N=44)$. The masses of ${ }^{85} \mathrm{Mo}$ and ${ }^{87} \mathrm{Tc}$ have then been measured at SHIPTRAP and big deviations of 1590(280) keV and 1430(300) keV from AME03 have been found [115]. When the SHIPTRAP values are adopted, the $S_{2 \mathrm{p}}$ values follow largely the systematic trend (see Fig. 8).

The strong deviations between AME03 and new mass measurements in this region, and in particular the observed dramatic change in systematic trends and the disappearance of irregularities when using new Penning trap masses casts doubt on the remaining AME03 masses and the resulting AME03 $S_{2 \mathrm{p}}$ trends in this region. More mass measurements towards more exotic isotones are urgently needed to verify or correct the AME03 data. Such measurements would also be important to show whether the linear trend in $S_{2 \mathrm{p}}$ values continues or whether there are indications of deformation. For example, in-beam gamma spectroscopy experiments have shown that almost all nuclei from krypton $(Z=36)$ to niobium $(Z=41)$ have permanent deformation when $N<44$ [116].

Mass measurements around ${ }^{100} \mathrm{Sn}$ offer a possibility to determine the $Z=50$ proton shell-gap energy $E_{\text {gap }, Z=50}$ from two-proton binding energies: $E_{\text {gap }, \mathrm{Z}=50}=S_{2 \mathrm{p}}(Z=$ $50)-S_{2 \mathrm{p}}(Z=52)$ (see Fig. 9). Penning trap mass measurements performed at JYFLTRAP $[3,41]$, SHIPTRAP [90], and ISOLTRAP [17] improve the precisions of the shell-gap energies a lot and reveal deviations from the 


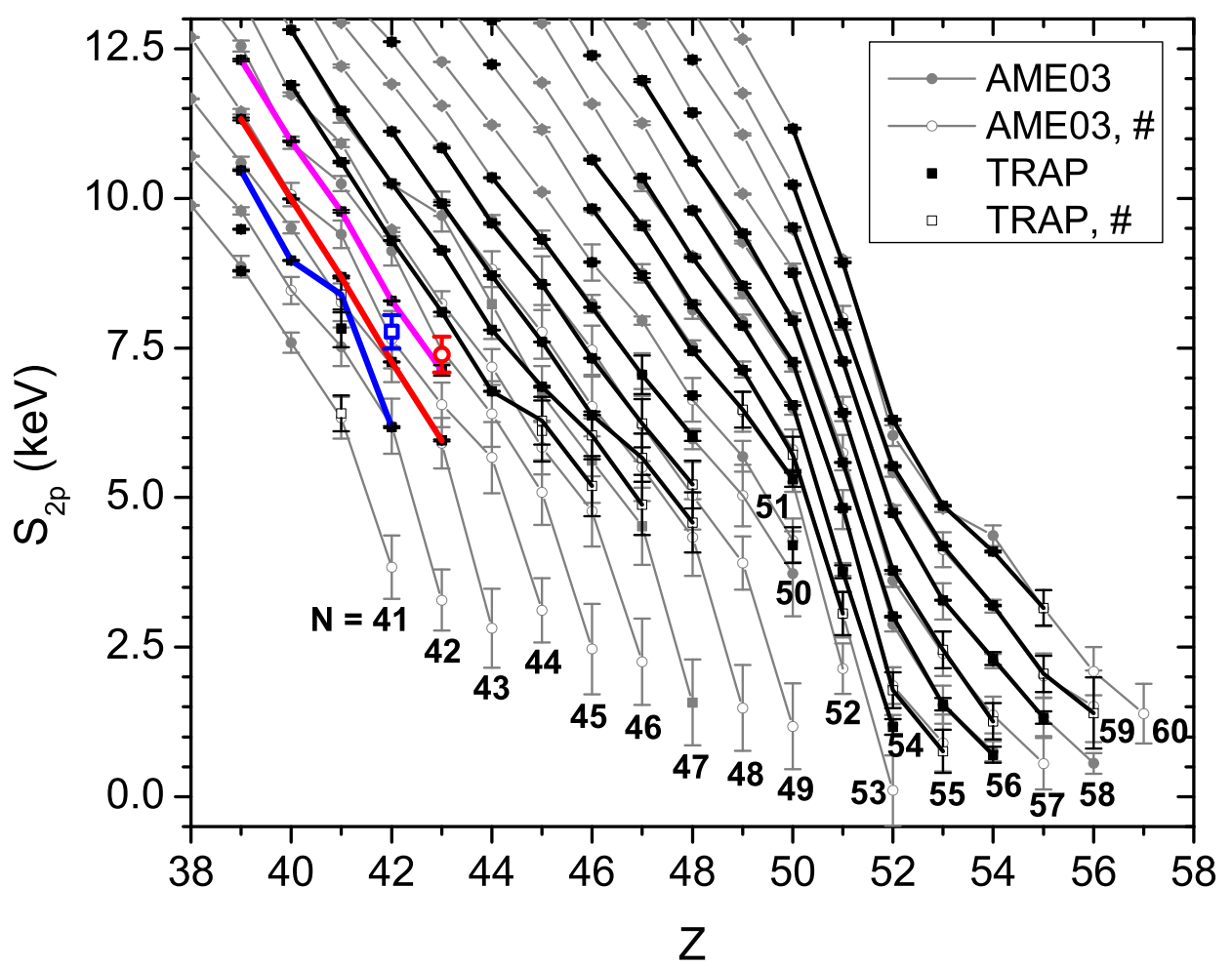

Fig. 8. (Color online) Two-proton separation energies determined at JYFLTRAP and comparison to the AME03 values [52]. The data from SHIPTRAP [90,115] and ISOLTRAP [17] have been taken into account in the Penning-trap data whenever possible. The 'TRAP' data cover the $S_{2 \mathrm{p}}$ values where at least one data set comes from a Penning-trap measurement and the missing data have been adopted from AME03. The 'AME03' values are based only on AME03. '\#' marks that the used AME03 value is based on extrapolations. The isotonic chains of $N=43, N=44$, and $N=45$ are shown in blue, red, and magenta, respectively. The open blue and red squares show the $S_{2 \mathrm{p}}$ values for ${ }^{85} \mathrm{Mo}$ and ${ }^{87} \mathrm{Tc}$ based on the JYFLTRAP and (erroneous) AME03 values.

AME03 values at $N=56-60$. With the new trap data a minimum in the shell-gap energies is achieved at $N=59$, and it is broader than with the AME03 data where a deeper minimum at $N=58$ is observed. Both data show an increasing shell-gap energy when proceeding towards the magic neutron number $N=50$.

\section{Astrophysical aspects}

\subsection{Nucleosynthesis in novae}

Novae occur in binary systems consisting of a white dwarf accreting hydrogen-rich matter from a main-sequence companion. Thermonuclear explosions of the accreted envelope drive the nova phenomenon. Novae on particularly massive oxygen-neon white dwarfs (ONe novae) are thought to reach peak temperatures of up to $4 \times 10^{8} \mathrm{~K}$. Under these conditions the explosive hydrogen burning occurs as a sequence of proton captures and $\beta^{+}$decays (and sometimes $(p, \alpha)$ reactions) proceeding up to $A=40$ due to the presence of NeNa-MgAl seed nuclei (see Refs. [10$13,117]$ ). Of special interest in ONe novae is the production of ${ }^{22} \mathrm{Na}$ and ${ }^{26} \mathrm{Al}$, which are sufficiently long-lived radioactive isotopes for their decay $\gamma$-radiation to be potentially observable. ${ }^{22} \mathrm{Na}\left(T_{1 / 2}=2.6019(4)\right.$ y [118] $)$ de- cays into a short-lived excited state of ${ }^{22} \mathrm{Ne}$ which deexcites to its ground state by emitting a $1.275 \mathrm{MeV} \gamma$-ray. Although several attempts to observe these $\gamma$-rays from nearby novae have been made, only an upper limit of the ejected ${ }^{22} \mathrm{Na}$ has been obtained [117]. The ${ }^{26} \mathrm{Al}$ ground state $\left(T_{1 / 2}=7.17(24) \times 10^{5}\right.$ y [118]) decays to an excited state of ${ }^{26} \mathrm{Mg}$ at $1.809 \mathrm{MeV}$. The $\gamma$-rays following the de-excitation of this state have been observed with $\gamma$-ray telescopes but the half-life is too long to associate them with a particular astrophysical event. The general distribution of Galactic $1.809 \mathrm{MeV}$ activity indicates an origin mainly associated with massive stars, but it is important to determine a possible nova contribution.

${ }^{22} \mathrm{Na}$ is produced in a so-called $\mathrm{NeNa}$ cycle where ${ }^{20} \mathrm{Ne}(p, \gamma){ }^{21} \mathrm{Na}$ is followed either by proton capture ${ }^{21} \mathrm{Na}(p, \gamma){ }^{22} \mathrm{Mg}\left(\beta^{+}\right){ }^{22} \mathrm{Na}$ or $\beta$ decay ${ }^{21} \mathrm{Na}\left(\beta^{+}\right){ }^{21} \mathrm{Ne}(p, \gamma)$ ${ }^{22} \mathrm{Na}\left(\beta^{+}\right)^{22} \mathrm{Ne}(p, \gamma){ }^{23} \mathrm{Na}(p, \alpha)^{20} \mathrm{Ne}$. In order to model the production of ${ }^{22} \mathrm{Na}$, the destruction channels, such as ${ }^{22} \mathrm{Mg}(p, \gamma){ }^{23} \mathrm{Al}$ and ${ }^{22} \mathrm{Na}(p, \gamma){ }^{23} \mathrm{Mg}$ have to be known precisely. At JYFLTRAP, ${ }^{23} \mathrm{Al}$ was found to be $22(19) \mathrm{keV}$ more proton-bound [26] than in AME03 [52]. Thus, the resonant contribution for the rate of ${ }^{22} \mathrm{Mg}(p, \gamma){ }^{23} \mathrm{Al}$ is little higher, and ${ }^{23} \mathrm{Al}$ is more resilient to destruction through photodissociation. Also the mass of ${ }^{23} \mathrm{Mg}$ has been measured at JYFLTRAP [26] but its impact on the calculated 
resonant rate for the ${ }^{22} \mathrm{Na}(p, \gamma)^{23} \mathrm{Mg}$ reaction has not been investigated.

${ }^{26} \mathrm{Al}$ is produced in a so-called $\mathrm{MgAl}$ cycle: ${ }^{24} \mathrm{Mg}(p, \gamma){ }^{25} \mathrm{Al}\left(\beta^{+}\right){ }^{25} \mathrm{Mg}(p, \gamma){ }^{26} \mathrm{Al}_{\text {g.s. }}\left(\beta^{+}\right){ }^{26} \mathrm{Mg}(p, \gamma){ }^{27} \mathrm{Al}$ $(p, \alpha)^{24} \mathrm{Mg}$. The production of ${ }^{26} \mathrm{Al}_{\text {g.s }}$. can be bypassed via ${ }^{25} \mathrm{Al}(p, \gamma)^{26} \mathrm{Si}\left(\beta^{+}\right)^{26} \mathrm{Al}^{m}\left(\beta^{+}\right)^{26} \mathrm{Mg}$. Therefore, the rate for the proton capture reaction ${ }^{25} \mathrm{Al}(p, \gamma){ }^{26} \mathrm{Si}$ is extremely important to constrain the model [117]. The JYFLTRAP mass value of ${ }^{26} \mathrm{Si}$ results in a $3.7(31) \mathrm{keV}$ lower proton separation energy for ${ }^{26} \mathrm{Si}$ than in AME03 [52]. This changes the calculated stellar reaction rates of ${ }^{25} \mathrm{Al}(p, \gamma){ }^{26} \mathrm{Si}$ by about $10 \%$ [33] compared to the rates calculated with the values from Ref. [119].

The reaction ${ }^{30} \mathrm{P}(p, \gamma){ }^{31} \mathrm{~S}$ plays a crucial role governing the flow towards ${ }^{32} \mathrm{~S}$ and heavier species in novae $[11,117]$. At ${ }^{30} \mathrm{P}$, the reaction flow has to proceed either via ${ }^{30} \mathrm{P}(p, \gamma){ }^{31} \mathrm{~S}(p, \gamma){ }^{32} \mathrm{Cl}\left(\beta^{+}\right){ }^{32} \mathrm{~S}$ or via ${ }^{30} \mathrm{P}(p, \gamma){ }^{31} \mathrm{~S}\left(\beta^{+}\right)$ ${ }^{31} \mathrm{P}(p, \gamma){ }^{32} \mathrm{~S}$. The ${ }^{30} \mathrm{P}(p, \gamma){ }^{31} \mathrm{~S}$ rate also has an effect on the ${ }^{30} \mathrm{Si}$ abundance [11]. The lower the proton capture rate, the more favorable is the $\beta^{+}$decay of ${ }^{30} \mathrm{P}$ and more ${ }^{30} \mathrm{Si}$ is produced. A more accurate reaction rate and ${ }^{30} \mathrm{Si}$ abundance (or ${ }^{30} \mathrm{Si} /{ }^{28} \mathrm{Si}$ abundance ratio) helps in the identification of presolar grains with a possible nova origin [120]. The proton separation energy obtained at JYFLTRAP [27] is 2.1(16) keV lower than the adopted value [52]. Although the difference is quite small, it has an effect on the calculated reaction rate of ${ }^{30} \mathrm{P}(p, \gamma){ }^{31} \mathrm{~S}$ which has been studied for example in Refs. [121-124].

Although in both cases the impact on the reaction rates is small, and there are much larger uncertainties from poorly known excited states, the measurements are a step towards more reliable rates that will be of particular importance once resonance parameters have been measured in future experiments. More importantly however, precise masses are essential for carrying out planned direct reaction rate measurements at radioactive beam facilities. Such experiments inherently suffer from limited beam intensities and resonance energies must be known precisely to make experiments feasible.

\section{$4.2 \nu p$-process}

The astrophysical $\nu p$-process has been suggested to occur in supernovae and possibly in gamma-ray bursts where proton-rich ejecta are created by strong neutrino fluxes $[6,7]$. In principle it proceeds similarly to the $r p$-process as a sequence of proton captures and $\beta^{+}$decays but the the flow towards heavier elements is accelerated via fast $(n, p)$ reactions bridging the slow $\beta^{+}$decays along the path of the $r p$-process. The neutrons needed for the $(n, p)$ reactions are created by antineutrino absorptions on protons. Nuclei with mass numbers $A>64$ are produced in the $\nu p$-process, which has been proposed to be a candidate for the origin of solar abundances of light $p$ nuclei ${ }^{92,94} \mathrm{Mo}$ and ${ }^{96,98} \mathrm{Ru}[6]$.

The $\nu p$ process has been modeled with the JYFLTRAP mass values $[19,40]$ in Ref. [19]. The JYFLTRAP mass excess value for ${ }^{88} \mathrm{Tc}$ was found to be $1031(218) \mathrm{keV}$ higher than in AME03 [52]. The heavier ${ }^{88} \mathrm{Tc}$ means a lower proton separation energy for ${ }^{88} \mathrm{Tc}$, which increases the reaction rate of ${ }^{88} \mathrm{Tc}(\gamma, p)^{87} \mathrm{Mo}$ and suppresses the flow through ${ }^{87} \mathrm{Mo}(p, \gamma){ }^{88} \mathrm{Tc}$. The main reaction flow to nuclei with $A>88$ proceeds then through ${ }^{87} \mathrm{Mo}(n, p){ }^{87} \mathrm{Nb}(p, \gamma)$ ${ }^{88} \mathrm{Mo}(p, \gamma)^{89} \mathrm{Tc}$. The stronger reaction flow through ${ }^{87} \mathrm{Mo}(n, p){ }^{87} \mathrm{Nb}$ and a higher abundance of ${ }^{87} \mathrm{Nb}$ enables also a flow through ${ }^{87} \mathrm{Nb}(n, p){ }^{87} \mathrm{Zr}$ which is seen in the abundance pattern as increased abundance of ${ }^{87} \mathrm{Sr}[19]$.

A recent mass measurement of ${ }^{87} \mathrm{Mo}$ [115] revealed a deviation of $810(220) \mathrm{keV}$ to the AME03 value. With the new mass values for ${ }^{87} \mathrm{Mo}[115]$ and ${ }^{88} \mathrm{Tc}$ [19], the proton separation energy of ${ }^{88} \mathrm{Tc}$ is in agreement with the AME03 value: the difference is $220(320) \mathrm{keV}$. This obviously changes the modeling results and further demonstrates the importance of accurate mass measurements.

Another example for the impact of the new mass values is ${ }^{90} \mathrm{Tc}$. The average mass measured at JYFLTRAP and SHIPTRAP [19] is $486(240) \mathrm{keV}$ higher than the AME03 value based on the $\beta$-decay energies. A lower proton separation energy increases the reaction rate of ${ }^{90} \mathrm{Tc}(\gamma, p)^{89} \mathrm{Mo}$ slightly. This, in turn, shifts some abundance from the $A=90$ chain into the $A=89$ chain and shows up in the abundance pattern as a decrease for ${ }^{90} \mathrm{Zr}$ [19].

The $\nu p$-process has been proposed to be a candidate for the origin of the solar abundances of the light $p$ nuclei ${ }^{92,94} \mathrm{Mo}$ and ${ }^{96,98} \mathrm{Ru}[6]$. Of special interest is the relative production of ${ }^{92} \mathrm{Mo}$ and ${ }^{94} \mathrm{Mo}$ governed by the proton separation energy of ${ }^{93} \mathrm{Rh}$. The more proton-bound ${ }^{93} \mathrm{Rh}$, the more ${ }^{94} \mathrm{Pd}$, and in the end, more ${ }^{94}$ Mo will be produced. In order to obtain the observed solar abundance ratio of ${ }^{92} \mathrm{Mo}$ and ${ }^{94} \mathrm{Mo}$ in current $\nu p$-process models it has been suggested that the proton separation energy of ${ }^{93} \mathrm{Rh}$ should be very close to $1.65 \mathrm{MeV}$ [125]. The JYFLTRAP and SHIPTRAP mass measurements [19] yield a proton separation energy $S_{\mathrm{p}}=2001(5) \mathrm{keV}$ in agreement with the result from the Canadian Penning Trap $S_{\mathrm{p}}=2007(9) \mathrm{keV}$ [21]. However, the experimental results disagree with the value needed for solar ${ }^{92} \mathrm{Mo} /{ }^{94} \mathrm{Mo}$ ratio in supernova outflows. This suggests that supernova outflows do not exclusively produce both molybdenum isotopes or that the winds are qualitatively different from the current supernova models [125].

The impact on mass variations on the abundances and production path of the $\nu p$-process have been investigated in Ref. [20]. There, ${ }^{93} \mathrm{Pd},{ }^{100} \mathrm{Cd}$, and ${ }^{101} \mathrm{In}$ were found to have highest influence on the final abundances and path. In addition, varying the mass excess of ${ }^{80} \mathrm{Zr}$ by $2 \sigma$ has a huge impact on the $\nu p$-process modeling due to large experimental mass uncertainty of ${ }^{80} \mathrm{Zr}(1490 \mathrm{keV})$ [20]. These nuclides will be searched for at IGISOL-4.

\section{3 rp-process}

Explosive hydrogen burning at temperatures in excess of $10^{8} \mathrm{~K}$ via the $r p$-process was first introduced in Ref. [1]. An astrophysical site where such burning occurs are x-ray bursts and, to a limited extent, novae. In this $r p$-process $[1,2]$, the rapid proton captures on seed nuclei or on the 
products of helium burning lead to production of heavier elements. Proton captures will proceed until they are inhibited by a low or negative $Q$ value, which leads to substantial $(\gamma, \mathrm{p})$ photodisintegration. At those points, the $r p$ process has to wait for a much slower $\beta^{+}$decay to happen. If the half-life of the $\beta$-decaying nucleus is particularly long the nucleus is called a waiting point.

Nuclear masses are relevant for the modeling of the $r p$-process due to the exponential dependence of effective waiting-point lifetimes on binding energy differences. The isotonic abundance ratios are exponentially dependent on proton separation energies as shown by the Saha equation (see e.g. Ref. [16]). The impact of mass uncertainties on the $r p$-process has been studied for example in Refs. [15, 20]. In Ref. [15], a list of nuclides whose masses should be measured in order to more reliably model the $r p$-process in $\mathrm{x}$-ray bursts was given. From that list, the masses of ${ }^{106} \mathrm{Sb}$ and ${ }^{107} \mathrm{Sb}$ have already been determined at JYFLTRAP [3], and interesting (but also experimentally challenging) candidates for future mass measurements at JYFLTRAP are ${ }^{31} \mathrm{Cl},{ }^{56} \mathrm{Cu},{ }^{61} \mathrm{Ga},{ }^{83,84} \mathrm{Nb},{ }^{86} \mathrm{Tc},{ }^{89} \mathrm{Ru},{ }^{90} \mathrm{Rh},{ }^{99} \mathrm{In}$, ${ }^{96} \mathrm{Ag},{ }^{97} \mathrm{Cd}$, and ${ }^{103} \mathrm{Sn}$.

The impact of mass variations on the light-curve, the final abundances (ashes) and path of the $r p$-process in $\mathrm{x}$ ray burst was modeled in Ref. [20]. ${ }^{94} \mathrm{Ag},{ }^{93} \mathrm{Pd}$, and ${ }^{91} \mathrm{Rh}$ were found to have highest influence on the nucleosynthesis modeling. In particular, the reaction ${ }^{93} \mathrm{Pd}(p, \gamma){ }^{94} \mathrm{Ag}$ has the strongest effect on the simulation of x-ray burst lightcurve. The sensitivity of the flow ratio between ${ }^{93} \mathrm{Pd}$ and ${ }^{94} \mathrm{Ag}$ is an indication of forward and backward flows of similar order of magnitude, and thus, suggests that ${ }^{93} \mathrm{Pd}$ is a waiting point [20]. JYFLTRAP aims to measure the masses of ${ }^{93} \mathrm{Pd}$ and ${ }^{94} \mathrm{Ag}$ in future.

\subsubsection{Doubly-magic waiting point ${ }^{56} \mathrm{Ni}$}

The typical duration of an x-ray burst is $10-100 \mathrm{~s} .{ }^{56} \mathrm{Ni}$ decays under terrestrial conditions mainly by electron capture. Electron densities during the $r p$-process are much smaller than in atoms, leading to a very long half-life of the order of at least $10 \mathrm{~h}[126,127]$ making ${ }^{56} \mathrm{Ni}$ essentially stable for typical $r p$-process timescales. Because of its doubly magic character the proton capture $Q$ value of ${ }^{56} \mathrm{Ni}$ is relatively low, making it a potentially unique $r p$-process waiting point that cannot be overcome by $\beta^{+}$ decay. Indeed, historically ${ }^{56} \mathrm{Ni}$ was considered as an endpoint of the $r p$-process [1]. However, with modern nuclear masses the proton capture $Q$ value is sufficienly high that ${ }^{56} \mathrm{Ni}$ only becomes a waiting point for temperatures in excess of around 2 GK $[2,128]$. Nevertheless, such temperatures are reached in some x-ray burst models, leading to a temporary stalling of the $r p$-process. The onset of processing beyond ${ }^{56} \mathrm{Ni}$ during the cooling of the burst depends sensitively on the exact $Q$ value for the ${ }^{56} \mathrm{Ni}$ proton capture. This $Q$ value has been directly measured at JYFLTRAP by using ${ }^{56} \mathrm{Ni}$ as a reference for ${ }^{57} \mathrm{Cu}$ in the mass measurement. In this way, the accuracy of the proton-capture $Q$ value was improved from $695(19) \mathrm{keV}$ [52] to $689.69(51) \mathrm{keV}[38]$.
Since the calculated resonant reaction rate to a state at an energy $E_{\mathrm{x}}$ is exponentially dependent on the resonance energies $E_{\mathrm{r}}=E_{\mathrm{x}}-Q_{\mathrm{p}, \gamma}$, the uncertainty of the proton capture $Q$ value has a large effect on the uncertainty of the ${ }^{56} \mathrm{Ni}(\mathrm{p}, \gamma)$ reaction rate. With the new $Q$ value, a factor of four in the uncertainty of the reaction rate at temperatures around 1 GK shown in Ref. [128] is removed and the new rate is a little higher than the one calculated with the old $Q$ value. The new $Q$ value supports the conclusions of Ref. [128] that the lifetime of ${ }^{56} \mathrm{Ni}$ against proton capture is much shorter than in the previous works. This reduces the minimum temperature required for the $r p$-process to proceed beyond ${ }^{56} \mathrm{Ni}$. This temperature threshold coincides with the temperature for the break out of the hot CNO cycles with the rates of Ref. [128]. Therefore, the rp-process can always proceed beyond ${ }^{56} \mathrm{Ni}$ provided sufficient amount of hydrogen are present.

\subsubsection{Quenching of the SnSbTe cycle}

The JYFLTRAP value for the proton separation energy of ${ }^{106} \mathrm{Sb}, S_{\mathrm{p}}=424(8) \mathrm{keV}$, disagrees considerably with the value $S_{\mathrm{p}}=930(210) \mathrm{keV}$ [129] based on the alphadecay energies and the mass of ${ }^{114} \mathrm{Cs}$ determined from its $\beta$-delayed proton decay. The value of Ref. [129] has been considered erroneous already in AME03 [52], where an extrapolated value of $S_{\mathrm{p}}=360(320) \# \mathrm{keV}$ agreeing with JYFLTRAP is given for ${ }^{106} \mathrm{Sb}$. The consequences of the new proton separation energies of ${ }^{106} \mathrm{Sb}$ and other nuclides in the SnSbTe region at JYFLTRAP, have been investigated in Ref. [3] with the same one-zone model as in Ref. [130]. In Ref. [130], ${ }^{105} \mathrm{Sb}$ was found proton unbound with $S_{\mathrm{p}}\left({ }^{105} \mathrm{Sb}\right)=-356(22) \mathrm{keV}$ based on $\alpha$-decay energies and the dependence of the branching into the SnSbTe cycle was plotted against the proton decay $Q$ value. Due to proton-unbound ${ }^{104,105} \mathrm{Sb}$ the $r p$-process has to proceed along the tin isotopes to ${ }^{105} \mathrm{Sn}$. There, the proton capture probability depends on its $Q_{(p, \gamma)}$ value, in other words, on the proton separation energy of ${ }^{106} \mathrm{Sb}$. With the new JYFLTRAP value for $S_{\mathrm{p}}\left({ }^{106} \mathrm{Sb}\right)$, only $3 \%$ of the reaction flow branches into the SnSbTe cycle at ${ }^{105} \mathrm{Sn}$, thus considerably attenuating the cycling via the chain ${ }^{105} \mathrm{Sn}-{ }^{106} \mathrm{Sb}-{ }^{107} \mathrm{Te}-{ }^{103} \mathrm{Sn}-{ }^{103} \mathrm{In}-{ }^{104} \mathrm{Sn}-{ }^{104} \mathrm{In}-{ }^{105} \mathrm{Sn}$.

The proton-separation energy of ${ }^{107} \mathrm{Sb}$ is also quite low, $588(7) \mathrm{keV}$, and only $13 \%$ branching to the SnSbTe cycle is found at ${ }^{106} \mathrm{Sn}$. Since the $\beta$-decay half-life of ${ }^{106} \mathrm{Sn}$ is long (2.1 min), it inhibits further processing towards ${ }^{107} \mathrm{Sn}$ and ${ }^{108} \mathrm{Sb}$, although the proton separation energy of ${ }^{108} \mathrm{Sb}(1222(8) \mathrm{keV})$ would be high enough for branching into the SnSbTe cycle.

Previous $r p$-process calculations had assumed there is a strong SnSbTe cycle where proton captures on ${ }^{105} \mathrm{Sn}$ and ${ }^{106} \mathrm{Sb}$ lead to ${ }^{107} \mathrm{Te}$, which then $\alpha$ decays back to ${ }^{103} \mathrm{Sn}$ creating helium and cycling the matter between $\mathrm{Sn}, \mathrm{Sb}$ and Te isotopes. This cycle resulted in a large accumulation of the longest-lived isotope in the SnSbTe cycle, ${ }^{104} \mathrm{Sn}$. This was also seen in the final composition of the burst ashes where ${ }^{104} \mathrm{Pd}$ was the most abundant element. 
With the new JYFLTRAP mass values the SnSbTe cycle develops closer to stability and requires therefore longer processing to be reached. Model calculations indicate that even under the most favorable conditions it is unlikely that a substantial SnSbTe cycle can develop in an $\mathrm{x}$-ray burst. As a result, the final composition of the ashes is characterized by a much broader distribution of ${ }^{68} \mathrm{Zn}$, ${ }^{72} \mathrm{Ge},{ }^{104} \mathrm{Pd},{ }^{105} \mathrm{Pd}$, and residual helium with comparable abundances. The absence of a strong SnSbTe cycle also reduces late-time ${ }^{4} \mathrm{He}$ production, which reduces the latetime boost of hydrogen consumption and the associated rise in energy production. This leads to slightly longer, less luminous tail. However, the effect is small since the Sn isotopes are reached at a very late stage in the burst. The quenching of the SnSbTe cycle leads also to a reduction of residual ${ }^{4} \mathrm{He}$ and ${ }^{12} \mathrm{C}$.

Although the masses needed to constrain the reaction flow in the SnSbTe cycle are now mostly well-known, uncertainties remain in the proton-capture rates on the antimony isotopes, in particular ${ }^{105} \mathrm{Sb}$ and ${ }^{106} \mathrm{Sb}$. It has been estimated that the proton-capture rate uncertainties vary up to a factor of 3 close to stability and the uncertainties far from stability might be larger [131]. For example, the ${ }^{106} \mathrm{Sb}(p, \gamma)$ rate calculated with the code from Ref. [132] is about a factor of 4 larger than the NON-SMOKER [133] rate used in Ref. [3].

\subsection{3 rp-process modeling with updated masses}

To explore the impact of recent Penning trap mass measurements, including the JYFLTRAP measurements, on the $r p$-process in x-ray bursts, and to explore the impact of remaining uncertainties, we carried out model calculations with the one-zone $\mathrm{x}$-ray burst model from $[20,134]$. Models employing the one-zone approximation reproduce the composition of the burst ashes and some general features of the burst light curve quite well, and at the same time allow to explore the impact of variations in the nuclear physics input in a computationally efficient way. The impact of mass uncertainties on x-ray burst models has been studied before using the post-processing approximation [15], which neglects the impact of modified nuclear physics on energy generation. Other work has been based on the same model employed here, but masses were varied within quoted 1- $\sigma$ errors in a random way [20].

Here we choose a different approach with the goal to illustrate the maximum possible impact that mass uncertainties can have on burst model predictions. To that end, we carry out two calculations for each set of masses, one where all proton capture $Q$ values are simultaneously increased by $3 \sigma$, and one where they are all simultaneously decreased by $3 \sigma .3 \sigma$ might seem like a large variation. However, for the Penning-trap mass measurements, error bars are so small that even a $3 \sigma$ variation has no significant effect on the model. On the other hand, for theoretically predicted masses, for example from Coulomb shifts, or for masses determined with other experimental methods, such as $\beta$-endpoint measurements, systematic errors make such large deviations more likely than they might appear based on a Gaussian probability distribution. In addition, the effects of masses on the rp-process can be highly nonlinear. Changes in the observables for $1 \sigma$ mass variations can therefore not simply be scaled to estimate the impact of large variations due to systematic errors. Previous sensitivity studies therefore likely underestimated the impact of mass uncertainties and have probably not identified all critical masses. Our study is therefore complementary to previous approaches - while it is not intended to provide statistically correct error bars for observables, it will identify all possible mass uncertainties and provide an envelope for observables that takes into account non-linearities and the possibility of systematic, non-Gaussian errors.

We ran calculations with three sets of masses: AME03, AME10, and NOJYFL. AME03 includes experimental and extrapolated masses from AME03, and uses Coulomb Shifts [135] to calculate the masses of more exotic nuclei with $Z>N$. AME10 uses in addition all Penning trap mass measurements in the $r p$-process region that have been published through 2010 (see Fig. 1). Recently published results $[79,115,136]$ were not included in the calculations. NOJYFL is similar to AME10 but we removed all masses that have been measured by JYFLTRAP to illustrate the impact of the JYFLTRAP program.

The $r p$-process reaction paths for the AME10 upper and lower proton capture $Q$ values are shown in Fig. 10 . Clearly, current mass uncertainties still allow for very large changes in the reaction paths. For the low $Q$ values the path below ruthenium is shifted by one mass unit, above ruthenium by 2 mass units closer to stability. With the low $Q$ values the ${ }^{80} \mathrm{Zr}$ waiting point is completely bypassed as the reaction flow proceeds towards stability in the yttrium isotopic chain. While JYFLTRAP mass measurements have made the formation of a SnSbTe cycle much more unlikely, the high $Q$-value calculation does develop such a cycle at ${ }^{103} \mathrm{Sn}$ because of the large uncertainties $\left(300 \# \mathrm{keV}\right.$ and $360 \# \mathrm{keV}$ ) that still exist for the ${ }^{103} \mathrm{Sn}$ and ${ }^{104} \mathrm{Sb}$ masses. While a significantly proton bound ${ }^{104} \mathrm{Sb}$, which would be required to form a SnSbTe cycle, seems unlikely given the trend of the measured proton separation energies of less neutron-deficient $\mathrm{Sb}$ isotopes, tentative experimental results indeed hint at ${ }^{104} \mathrm{Sb}$ not being a fast proton emitter [130].

Fig. 11 demonstrates that the burst light curve can be influenced dramatically by nuclear masses. At the extreme of very high $Q$ values, the major waiting points can be bypassed efficiently and processing towards heavy nuclei during the burst cooling phase when the luminosity is decreasing is greatly accelerated leading to a significant increase in energy generation, and fast exhaustion of fuel. The result is the development of a shoulder in the light curve during the early cooling phase, and a second peak in the burst profile after about $70 \mathrm{~s}$. On the other hand, very low $Q$ values increase photodisintegration, which hampers proton capture and results in a slower $r p$-process closer to stability. As a consequence, energy is generated at a reduced rate, but for a longer time leading to a very long burst tail lasting about 5 minutes past the initial burst peak. Clearly Penning trap mass measurements since 2003 


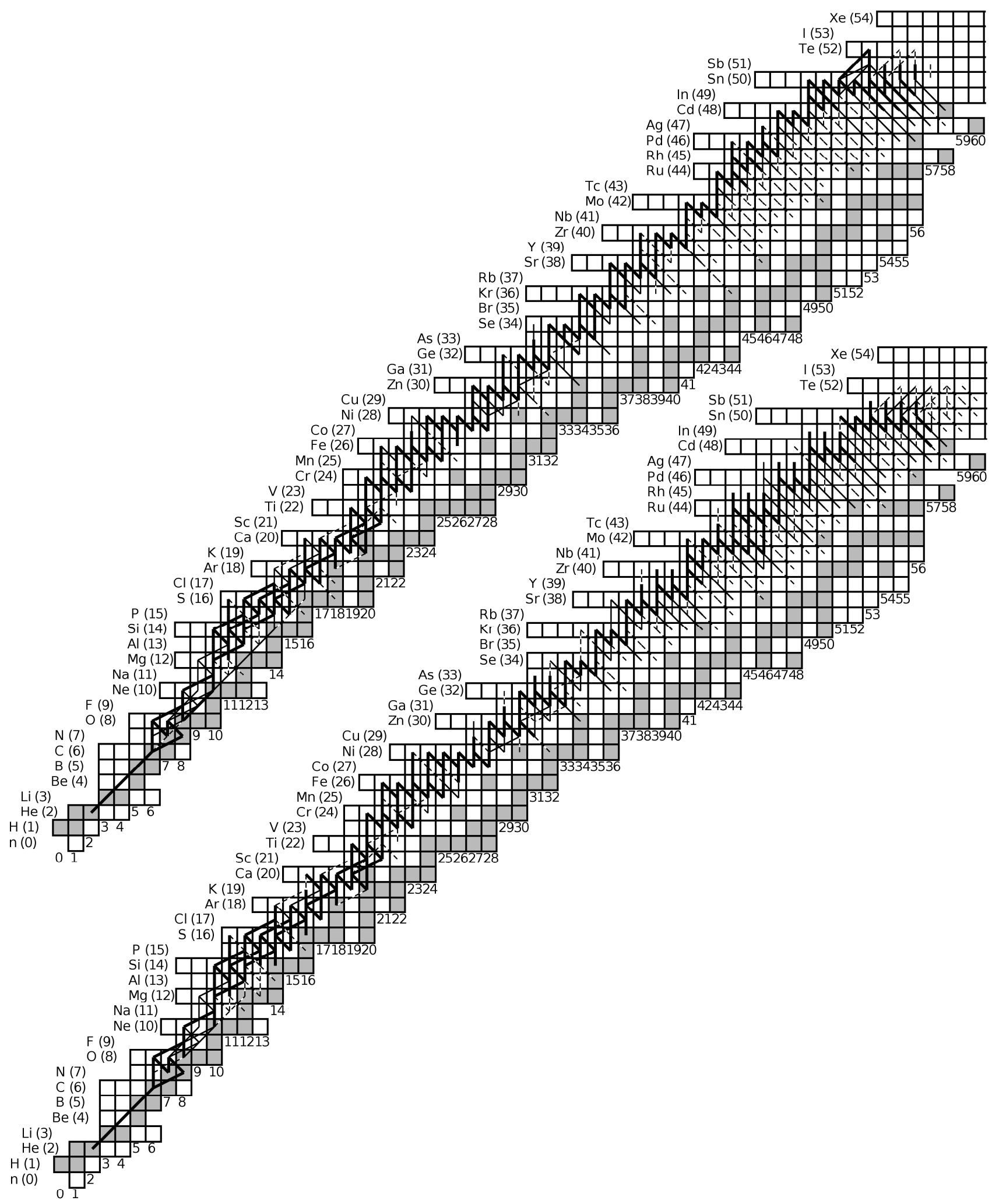

Fig. 10. The $r p$-process reaction flows corresponding the AME10 upper $(+3 \sigma)$ (top) and lower $(-3 \sigma)$ proton capture $Q$ values (bottom). Plotted are net flows of $>10 \%$ (thick solid line), 1\%-10\% (thin solid line), and $0.1 \%-1 \%$ (dashed line) of the flow through the $3 \alpha$ reaction. Note that flows for $(\mathrm{p}, \gamma)$ reactions are numerically not accurate for cases of $(\mathrm{p}, \gamma)-(\gamma, \mathrm{p})$ equilibrium near the proton drip line. 


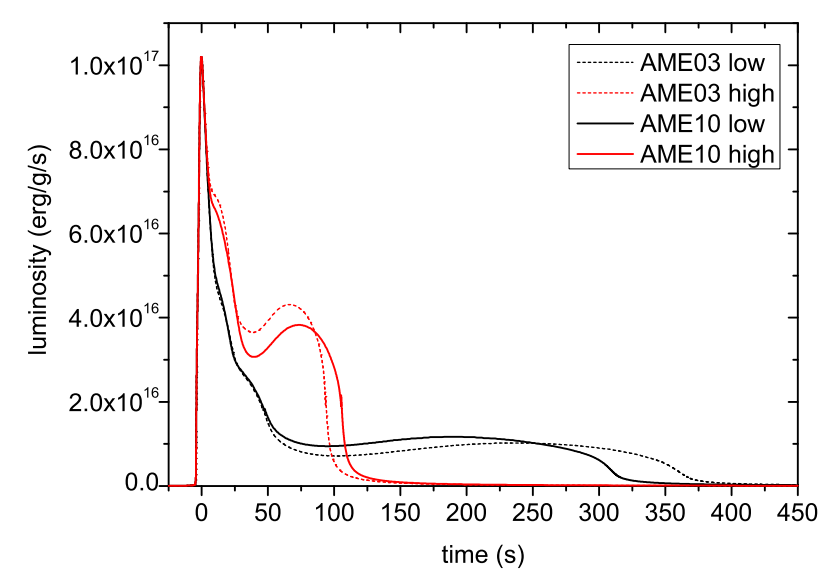

Fig. 11. (Color online) Calculated emitted luminosity as a function of time during an x-ray burst. The burst light curve is based on proton-capture $\mathrm{Q}$ values from (i) AME03 values varied $3 \sigma$ up (AME03 high, red dashed line) or down (AME03 low, black dashed line) and (ii) AME10 values (including all new Penning trap measurements before 2011) varied $3 \sigma$ up (AME10 high, red solid line) or down (AME10 low, black solid line).

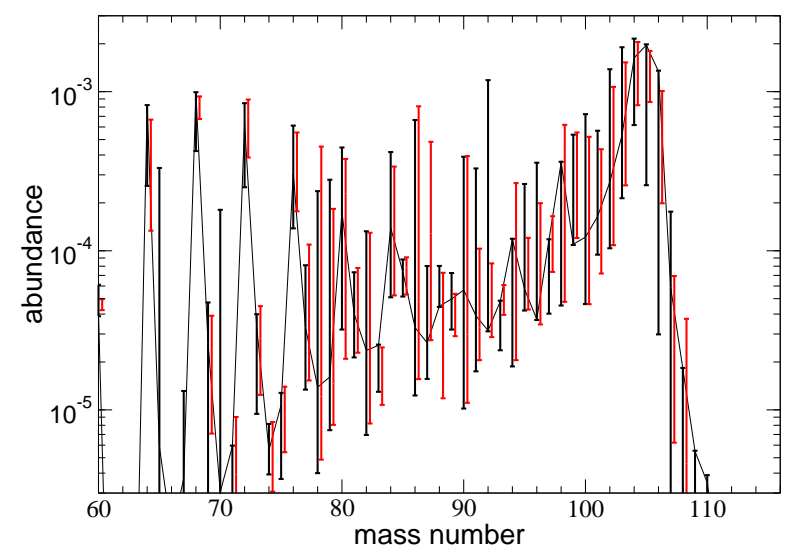

Fig. 12. (Color online) Final abundance (mass fraction divided by mass number) distribution as a function of mass number for an x-ray burst for data sets AME03 and AME10. The vertical bars represent the variation of the abundances obtained with upper $(+3 \sigma)$ and lower $(-3 \sigma)$ proton-capture $Q$ values. The left part of the data pairs belongs to AME03 (black) and the right part to AME10 (red).

have not yet reached the majority of $r p$-process masses and do reduce the uncertainty only somewhat. It should be noted that the systematic AME03 mass extrapolations play an important role in the model calculations. These extrapolations were based on the AME03 experimental data sets. Mass measurements since 2003 would likely lead to improved extrapolations and more reliable burst calculations. This effect is not included here, and its exploration needs to wait for the publication of the next Atomic Mass Evaluation.

Figs. 12 and 13 illustrate corresponding variations in the final composition of the ashes. The composition is given as a function of mass number, as the ashes decay

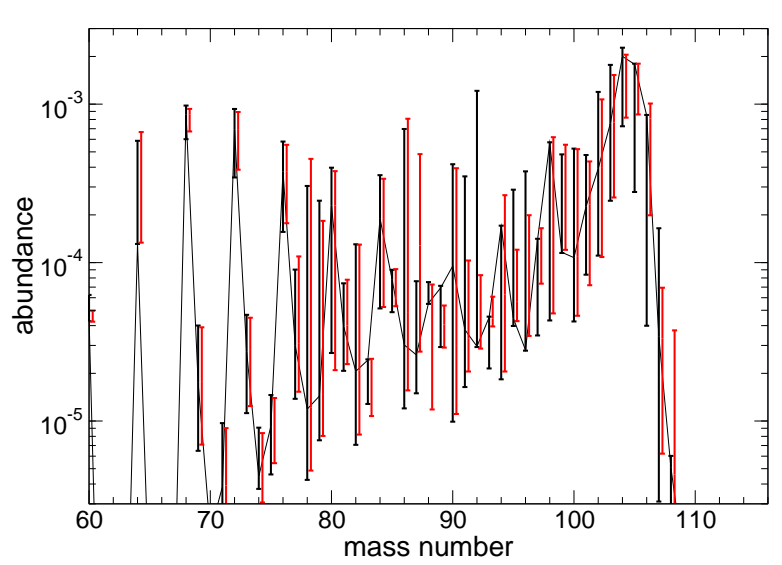

Fig. 13. (Color online) Same as Fig. 12 but for x-ray burst calculations with AME10 and NOJYFL. The left part of the data pairs belongs to NOJYFL (black) and the right part to AME10 (red).

along a mass chain until the first stable isotope is reached. Clearly significant variations up to an order of magnitude are possible, but not more. For many mass numbers, the maximum variations are much smaller. Among the important most abundant isotopes in the ashes, Penning trap mass measurements since 2003 had the largest impact on the $A=68,91,92,105,106$ isobars where uncertainties in the final abundance were drastically reduced (see Fig. 12). The JYFLTRAP mass measurements had a major impact on all of these, with the exception of $A=68$ (see Fig. 13). The reduction of the $A=92$ abundance uncertainty is particularly important in light of the interest in a possible production of ${ }^{92} \mathrm{Mo}$ in the $r p$-process.

\section{Outlook}

Mass measurements on neutron-deficient nuclides of astrophysical interest will continue with JYFLTRAP at the new IGISOL-4 facility. There will be more beam time available since the two cyclotrons, MCC-30 and K-130, can work in parallel. The new facility should also offer better and cleaner conditions for mass measurements. A permanent yield station and a post-trap spectroscopy setup will help in monitoring the experiments. Besides IGISOL4 there have been major developments in the JYFLTRAP mass measurements since the first measurements of $r p$ process nuclei [40]. A new fast cleaning procedure to produce isomerically pure ion samples has been developed [37]. The Ramsey method of time-separated oscillatory fields $[137,138]$ has been successfully applied to short-lived ions of astrophysical interest. Precise data on mass-dependent and residual uncertainties of JYFLTRAP have been obtained via carbon cluster measurements [48]. Interleavedly performed measurements [34] reduce the uncertainty due to temporal $B$-field fluctuations, which will result in a promising improvement on future mass measurements for neutron-deficient nuclei.

With the light-ion guide at IGISOL-4, nuclides important for modeling the explosive hydrogen burning in ONe 
novae as well as for testing the IMME, such as ${ }^{27} \mathrm{P},{ }^{31} \mathrm{Cl}$, and ${ }^{32} \mathrm{Cl}$, will be produced. Also the $Q_{\mathrm{EC}}$ value measurements of mirror nuclei will be continued. In the heavier mass region, proton or ${ }^{3} \mathrm{He}$ beams on a ${ }^{92}$ Mo target could help in populating the low-spin isomers in ${ }^{91} \mathrm{Tc}$ and ${ }^{93} \mathrm{Ru}$. More exotic species, such as the $N=50$ isotones ${ }^{93} \mathrm{Tc}$, ${ }^{97} \mathrm{Ag},{ }^{98} \mathrm{Cd},{ }^{99} \mathrm{In}$, and ${ }^{100} \mathrm{Sn}$ could be searched for with the heavy-ion guide. In addition, a mass measurement of the waiting-point nucleus ${ }^{80} \mathrm{Zr}$ would be very interesting. The masses of ${ }^{94} \mathrm{Ag}$ and ${ }^{93} \mathrm{Pd}$ have been shown to have a high impact on $r p$ process models thus should be measured. ${ }^{93} \mathrm{Pd}$ could be produced at HIGISOL whereas for ${ }^{94} \mathrm{Ag}$, a special hot cavity laser ion source has been developed [139]. Since the half-life of the ${ }^{94} \mathrm{Ag}$ ground-state is only around $30 \mathrm{~ms}$, it is too short-lived for measurements at JYFLTRAP. However, the much longer-lived isomeric states $\left(7^{+}, 0.55(6) \mathrm{s}[140]\right)$ and $\left(21^{+}, 0.40(4) \mathrm{s}[140]\right)$ could be measured. Of these, the $21^{+}$spin-gap isomer has gained much interest recently due to its claimed two-proton decay [141].

Taking into account all the developments carried out at JYFLTRAP and IGISOL, new mass measurements of $\mathrm{Y}, \mathrm{Nb}, \mathrm{Mo}$, and Tc isotopes at the heavy-ion ion guide at IGISOL-4 would be relevant. These measurements would confirm the nature of the measured state (ground state or isomer) in previous experiments $[19,40,115]$. In addition, the few nuclides $\left({ }^{92} \mathrm{Tc},{ }^{90} \mathrm{Ru},{ }^{107} \mathrm{Sb}\right.$, and $\left.{ }^{109} \mathrm{Sb}\right)$ for which deviations to the SHIPTRAP values have been found, could be reinvestigated.

More accurate mass values would be obtained via networks of mass measurements, where a single reference atom would not play such a big role. This would also give better information on the mass surface and be useful for mass predictions. In addition, direct frequency ratio measurements between proton-capture mother and daughter would result in more precise proton-capture $Q$ values. Identification of the ground and isomeric states and/or production ratios for possible isomer corrections should be investigated via post-trap spectroscopy. In many cases, simple half-life measurements based on $\beta$ particles would enlighten the situation a lot. In summary, there is a wealth of fascinating experiments on neutron-deficient nuclei of astrophysical interest to be performed with JYFLTRAP in future.

This work has been supported by the Academy of Finland under the Finnish Centre of Excellence Programme 2006-2011 (Nuclear and Accelerator Based Physics Programme at JYFL). A.K. acknowledges the support from the Academy of Finland under the project 127301.

\section{References}

1. R. K. Wallace and S. E. Woosley, Astrophys. J. Suppl. Ser. 45, 389 (1981).

2. H. Schatz et al., Phys. Rep. 294, 167 (1998).

3. V.-V. Elomaa et al., Phys. Rev. Lett. 102, 252501 (2009).

4. S. E. Woosley et al., Astrophys. J. Suppl. S. 151, 75 (2004).
5. J. José, F. Moreno, A. Parikh, and C. Iliadis, Astrophys. J. Suppl. S. 189, 204 (2010).

6. C. Fröhlich et al., Phys. Rev. Lett. 96, 142502 (2006).

7. J. Pruet et al., Astrophys. J. 644, 1028 (2006).

8. S. Wanajo, Astrophys. J. 647, 1323 (2006).

9. S. Wanajo, H.-T. Janka, and S. Kubono, Astrophys. J. 729, 46 (2011).

10. J. José, A. Coc, and M. Hernanz, Astrophys. J. 520, 347 (1999).

11. J. José, A. Coc, and M. Hernanz, Astrophys. J. 560, 897 (2001).

12. J. José and M. Hernanz, J. Phys. G 34, R431 (2007).

13. S. Starrfield et al., Astrophys. J. 692, 1532 (2009).

14. S. A. Glasner and J. W. Truran, Astrophys. J. Lett. 692, L58 (2009).

15. A. Parikh et al., Phys. Rev. C 79, 045802 (2009).

16. H. Schatz, Int. J. Mass Spectrom. 251, 293 (2006).

17. M. Breitenfeldt et al., Phys. Rev. C 80, 035805 (2009).

18. J. Savory et al., Phys. Rev. Lett. 102, 132501 (2009).

19. C. Weber et al., Phys. Rev. C 78, 054310 (2008).

20. T. Fleckenstein, Diploma thesis, Justus-LiebigUniversität Gießen, 2008.

21. J. Fallis et al., Phys. Rev. C 78, 022801 (2008).

22. G. Savard et al., Hyperfine Interact. 132, 221 (2001).

23. M. Mukherjee et al., Eur. Phys. J. A 35, 1 (2008).

24. R. Ringle et al., Nucl. Instrum. Methods Phys. Res. A 604, 536 (2009).

25. M. Block et al., Eur. Phys. J. D 45, 39 (2007).

26. A. Saastamoinen et al., Phys. Rev. C 80, 044330 (2009).

27. A. Kankainen et al., Phys. Rev. C 82, 052501 (2010).

28. T. Eronen, Ph.D. thesis, Department of Physics, University of Jyväskylä, 2008.

29. E. Wigner, Phys. Rev. 51, 947 (1937).

30. J. Äystö, Nucl. Phys. A 693, 477 (2001).

31. M. Oinonen et al., Nucl. Instrum. Methods Phys. Res. A 416, 485 (1998).

32. J. Huikari et al., Nucl. Instrum. Methods Phys. Res. B 222, 632 (2004).

33. T. Eronen et al., Phys. Rev. C 79, 032802 (2009).

34. T. Eronen et al., Phys. Rev. Lett. 103, 252501 (2009).

35. T. Eronen et al., Phys. Rev. Lett. 97, 232501 (2006).

36. T. Kurtukian Nieto et al., Phys. Rev. C 80, 035502 (2009).

37. T. Eronen et al., Phys. Rev. Lett. 100, 132502 (2008).

38. A. Kankainen et al., Phys. Rev. C 82, 034311 (2010).

39. T. Eronen et al., Phys. Lett. B 636, 191 (2006).

40. A. Kankainen et al., Eur. Phys. J. A 29, 271 (2006).

41. V. Elomaa et al., Eur. Phys. J. A 40, 1 (2009).

42. A. Nieminen et al., Nucl. Instrum. Methods Phys. Res. A 469, 244 (2001).

43. V. S. Kolhinen et al., Nucl. Instrum. Methods Phys. Res. A 528, 776 (2004).

44. G. Savard et al., Phys. Lett. A 158, 247 (1991).

45. G. Gräff, H. Kalinowsky, and J. Traut, Z. Phys. A 297, 35 (1980).

46. M. König et al., Int. J. Mass Spectrom. Ion Processes 142, 95 (1995).

47. A. Kellerbauer et al., Eur. Phys. J. D 22, 53 (2003).

48. V.-V. Elomaa et al., Nucl. Instrum. Methods Phys. Res. A 612, 97 (2009).

49. W. Benenson and E. Kashy, Rev. Mod. Phys. 51, 527 (1979). 
50. M. Bentley and S. Lenzi, Prog. Part. and Nucl. Phys. 59, 497 (2007).

51. N. Auerbach, Phys. Rep. 98, 273 (1983).

52. G. Audi, A. H. Wapstra, and C. Thibault, Nucl. Phys. A 729, 337 (2003).

53. R. Firestone, Nucl. Data Sheets 108, 1 (2007).

54. V. E. Iacob et al., Phys. Rev. C 74, 045810 (2006).

55. C. Wrede et al., Phys. Rev. C 81, 055503 (2010).

56. M. Bhattacharya et al., Phys. Rev. C 77, 065503 (2008).

57. M. Redshaw, J. McDaniel, and E. G. Myers, Phys. Rev. Lett. 100, 093002 (2008).

58. A. A. Kwiatkowski et al., Phys. Rev. C 80, 051302 (2009).

59. A. Paul, S. Röttger, A. Zimbal, and U. Keyser, Hyperfine Interact. 132, 189 (2001).

60. P. Rikmenspoel and D. V. Patter, Nucl. Phys. 24, 494 (1961).

61. G. Rickards, B. E. Bonner, and G. C. Phillips, Nucl. Phys. 86, 167 (1966).

62. R. Nussbaum et al., Physica 20, 571 (1954).

63. K. Sato, Mass Spectrom. (Japan) 5, 54 (1964).

64. S. Antman, H. Pettersson, and A. Suarez, Nucl. Phys. A 94, 289 (1967).

65. J. M. Freeman et al., Nucl. Phys. 65, 113 (1965).

66. B. E. Bonner, G. Rickards, D. L. Bernard, and G. C. Phillips, Nucl. Phys. 86, 187 (1966).

67. J. Overley, P. Parker, and D. Bromley, Nucl. Instrum. Methods 68, 61 (1969).

68. L. Erikson et al., Phys. Rev. C 81, 045808 (2010).

69. B. Sherrill et al., Phys. Rev. C 28, 1712 (1983).

70. M. B. Greenfield, C. R. Bingham, E. Newman, and M. J. Saltmarsh, Phys. Rev. C 6, 1756 (1972).

71. N. Severijns, M. Tandecki, T. Phalet, and I. S. Towner, Phys. Rev. C 78, 055501 (2008).

72. O. Naviliat-Cuncic and N. Severijns, Phys. Rev. Lett. 102, 142302 (2009).

73. H. Fujita et al., Phys. Rev. C 75, 034310 (2007).

74. C. Guénaut et al., Phys. Rev. C 75, 044303 (2007).

75. D. Rodríguez et al., Nucl. Phys. A 769, 1 (2006).

76. F. Herfurth et al., Eur. Phys. J. A 15, 17 (2002).

77. F. Herfurth et al., Nucl. Phys. A 746, 487 (2004), proceedings of the Sixth International Conference on Radioactive Nuclear Beams (RNB6).

78. G. Sikler et al., Nucl. Phys. A 763, 45 (2005).

79. F. Herfurth et al., Eur. Phys. J. A 47, 1 (2011).

80. P. Schury et al., Phys. Rev. C 75, 055801 (2007).

81. J. A. Clark et al., Phys. Rev. Lett. 92, 192501 (2004).

82. M. L. Commara et al., Nucl. Phys. A 708, 167 (2002).

83. C. Plettner et al., Nucl. Phys. A 733, 20 (2004).

84. K. Kaneko, Y. Sun, M. Hasegawa, and T. Mizusaki, Phys. Rev. C 77, 064304 (2008).

85. E. Nolte and H. Hick, Z. Phys. A 305, 289 (1982).

86. E. Bouchez et al., Phys. Rev. Lett. 90, 082502 (2003).

87. J. C. Hardy, L. C. Carraz, B. Jonson, and P. G. Hansen, Phys. Lett. B 71, 307 (1977).

88. S. Issmer et al., Eur. Phys. J. A 2, 173 (1998).

89. S. Kato et al., Phys. Rev. C 41, 1276 (1990).

90. A. Martín et al., Eur. Phys. J. A 34, 341 (2007).

91. J. A. Clark et al., in The 4th International Conference on Exotic Nuclei and Atomic Masses, edited by C. J. Gross, W. Nazarewicz, and K. P. Rykaczewski (Springer Berlin Heidelberg, ADDRESS, 2005), pp. 629-632.

92. G. Audi, O. Bersillon, J. Blachot, and A. H. Wapstra, Nucl. Phys. A 729, 3 (2003).
93. J. Döring, A. Aprahamian, and M. Wiescher, J. Res. Natl. Inst. Stand. Technol. 105, 43 (2000).

94. S. Dean et al., Eur. Phys. J. A 21, 243 (2004).

95. D. Kast et al., Z. Phys. A 356, 363 (1996).

96. A. Kankainen et al., Eur. Phys. J. A 25, 355 (2005).

97. A. H. Wapstra, G. Audi, and C. Thibault, Nucl. Phys. A 729, 129 (2003).

98. R. Barden et al., Z. Phys. A 329, 319 (1988).

99. H. Keller et al., Z. Phys. A 340, 363 (1991).

100. M. Kavatsyuk, L. Batist, M. Karny, and E. Roeckl, Int. J. Mass Spectrom. 251, 138 (2006).

101. A. Plochocki et al., Nucl. Phys. A 332, 29 (1979).

102. R. Kirchner et al., Phys. Lett. B 70, 150 (1977).

103. E. Roeckl et al., Phys. Lett. B 78, 393 (1978).

104. D. Schardt et al., Nucl. Phys. A 326, 65 (1979).

105. F. Heine et al., Z. Phys. A 340, 225 (1991).

106. D. Schardt et al., Nucl. Phys. A 368, 153 (1981).

107. R. J. Tighe et al., Phys. Rev. C 49, R2871 (1994).

108. D. D. Bogdanov, V. A. Karnaukhova, and L. A. Petrov, Yad. Fizika 17, 457 (1973).

109. Y. Litvinov et al., Nucl. Phys. A 756, 3 (2005).

110. K. Sharma et al., Proceedings of 9th Int. Conf. Atomic Masses and Fundamental constants AMCO-9 and 6th Int. Conf. Nuclei far from Stability NUFAST-6, p. 31, 1992.

111. M. G. Johnston et al., J. Phys. G 8, 1405 (1982).

112. F. Heine et al., Proc. of 9th Int. Conf. Atomic Masses and Fundamental constants AMCO-9 and 6th Int. Conf. Nuclei far from Stability NUFAST-6, p. 331, 1992.

113. R. D. Page et al., Phys. Rev. C 49, 3312 (1994).

114. T. Faestermann et al., Phys. Lett. B 137, 23 (1984).

115. E. Haettner et al., Phys. Rev. Lett. 106, 122501 (2011).

116. S. M. Fischer et al., Phys. Rev. C 75, 064310 (2007).

117. J. José, M. Hernanz, and C. Iliadis, Nucl. Phys. A 777, 550 (2006).

118. P. M. Endt, Nucl. Phys. A 633, 1 (1998).

119. A. Parikh et al., Phys. Rev. C 71, 055804 (2005).

120. S. Amari et al., Astrophys. J. 551, 1065 (2001).

121. D. G. Jenkins et al., Phys. Rev. C 73, 065802 (2006).

122. Z. Ma et al., Phys. Rev. C 76, 015803 (2007).

123. C. Wrede et al., Phys. Rev. C 76, 052802 (2007).

124. C. Wrede et al., Phys. Rev. C 79, 045803 (2009).

125. J. L. Fisker, R. D. Hoffman, and J. Pruet, Astrophys. J. Lett. 690, L135 (2009).

126. B. Sur et al., Phys. Rev. C 42, 573 (1990).

127. J. Lund Fisker, G. Martínez-Pinedo, and K. Langanke, Eur. Phys. J. A 5, 229 (1999).

128. O. Forstner et al., Phys. Rev. C 64, 045801 (2001).

129. A. Plochocki et al., Phys. Lett. B 106, 285 (1981).

130. C. Mazzocchi et al., Phys. Rev. Lett. 98, 212501 (2007).

131. W. Rapp et al., Astrophys. J. 653, 474 (2006).

132. M. Aikawa et al., Astron. Astrophys. 441, 1195 (2005).

133. T. Rauscher and F.-K. Thielemann, At. Data Nucl. Data Tables 79, 47 (2001).

134. H. Schatz et al., Phys. Rev. Lett. 86, 3471 (2001).

135. B. A. Brown et al., Phys. Rev. C 65, 045802 (2002).

136. X. L. Tu et al., Phys. Rev. Lett. 106, 112501 (2011).

137. S. George et al., Int. J. Mass Spectrom. 264, 110 (2007).

138. M. Kretzschmar, Int. J. Mass Spectrom. 264, 122 (2007).

139. M. Reponen et al., Eur. Phys. J. A 42, 509 (2009).

140. D. Abriola and A. Sonzogni, Nucl. Data Sheets 107, 2423 (2006).

141. I. Mukha et al., Nature 439, 298 (2006). 